\title{
TITLE:
}

\section{Nonrecourse financing and securitization}

AUTHOR(S):

Kobayashi, Mami; Osano, Hiroshi

CITATION:

Kobayashi, Mami ...[et al]. Nonrecourse financing and securitization. Journal of Financial Intermediation 2012, 21(4): 659-693

ISSUE DATE:

2012-10

URL:

http://hdl.handle.net/2433/160679

\section{RIGHT:}

C 2012 Elsevier Inc.; この論文は出版社版でありません。引用の際には 出版社版をご確認ご利用ください。; This is not the published version. Please cite only the published version. 


\title{
Nonrecourse Financing and Securitization*
}

\author{
Mami Kobayashi ${ }^{\dagger}$ and Hiroshi Osano
}

\author{
August 22, 2010 \\ First Revision: July 24, 2011 \\ Second Revision: November 25, 2011 \\ Final Revision: April 2, 2012
}

*The authors would like to thank Munetomo Ando, Hiroshi Fujiki, Tatsuya Kikutani, Yutaka Soejima, the Editor (Viral V. Acharya), an anonymous referee, and seminar and conference participants at the Bank of Japan, the Contract Theory Workshop, the Monetary Economics Workshop, the Research Institute of Economy, Trade \& Industry (RIETI), and the annual meeting of the Japan Association for Applied Economics for their helpful comments and suggestions on an earlier version of this paper, and Mamiko Terasaki for her research assistance. This research was supported financially by the Japan Society for the Promotion of Science under Grant No. (C) 20530152. The research is based partly on research supported by the Bank of Japan and undertaken while the second author was visiting the Bank of Japan. The views expressed herein are the authors' and do not reflect the views of the Bank of Japan.

Correspondence to: Hiroshi Osano, Institute of Economic Research, Kyoto University, Sakyo-ku, Kyoto 606-8501, Japan.

E-mail: osano@kier.kyoto-u.ac.jp; Tel: +81-75-753-7131; Fax: +81-75-753-7138.

${ }^{\dagger}$ Faculty of Economics, Kinki University

${ }^{\ddagger}$ Institute of Economic Research, Kyoto University 


\begin{abstract}
We consider the role of the nonrecourse financing of securitization by a financial institution (FI). Our model suggests that even though the FI has the opportunity to provide liquidity support afterward, it is optimal for the FI to use the nonrecourse financing of securitization initially, because the nonrecourse security makes liquidation of the original asset more attractive for an FI that knows that the original asset is bad. However, our model also predicts that the nonrecourse financing of securitization, together with short-term maturity financing, forces the financial system to perform inefficiently in handling troubled loans and causes problems with inefficient liquidity support and overinvestment under certain conditions, despite the nonrecourse property of securitization. The theoretical results provide empirical implications for recent problems with securitized and structured finance in the United States and Europe.
\end{abstract}

JEL Classification Code: D82, D86, G21, G23, G24, G33.

Keywords: asset-backed securities, financial distress, liquidity, maturity mismatch, structured finance. 


\section{Introduction}

Many commercial and investment banks have recently issued structured finance products such as mortgage-backed securities (MBSs) and collateralized debt obligations (CDOs) into which mortgages and other loans are pooled. These banks have also made extensive use of off-balance-sheet financing in originating these products. For example, the portfolios of structured finance products are transferred to off-balance-sheet vehicles. To raise funds for originating the products, banks act as sponsors for asset-backed commercial paper (ABCP) programs. In these programs, off-balance-sheet vehicles-ABCP conduits and structured investment vehicles (SIVs) - issue ABCP to third-party investors, and pay their sponsoring banks for transferred assets with the proceeds of the ABCP. The principal and interest payments of ABCP to third-party investors are made by the cash flows that are generated from the assets transferred to the off-balance-sheet vehicles. As a result, the off-balancesheet vehicles invest in illiquid long-maturity assets and issue short-maturity paper. Because the debt obligations of off-balance-sheet vehicles are usually contractually remote from the sponsoring banks, these banks are not required to treat the assets and debt obligations of such vehicles as their own. In this sense, such financing has the nonrecourse property for sponsoring banks. However, to ensure funding liquidity, sponsoring banks typically provide their conduits and vehicles with credit enhancement, liquidity support, or both, despite the nonrecourse property. For this reason, if an ABCP conduit or SIV cannot roll over its maturing paper, and thus liquidity drawdowns are made to the full extent of the outstanding commercial paper (CP), the sponsoring bank is forced to provide funding support to the ABCP conduit or SIV and thus becomes the sole funding provider. Note that renegotiation is practically impossible in securitization. The sponsoring bank can then direct the ABCP conduit or the SIV either to maintain its assets or to sell them in the market. ${ }^{1}$

The federal mortgage agencies (Fannie Mae and Freddie Mac) also issue MBSs and structured mortgage products. As they guarantee each MBS they issue, securitization involves a liquidity support problem whose effect is similar to that of the securitization of mortgages from commercial and investment banks. ${ }^{2}$

The goal of this paper is to explore the role of nonrecourse financing of securitization. The main question in this paper is whether it is optimal for a financial institution (FI) to

\footnotetext{
${ }^{1}$ For institutional details, see Gorton (2009), Brunnermeier (2009), and Coval, Jurek and Stafford (2009).

${ }^{2}$ For these issues, see the website of Freddie Mac (http://www.freddiemac.com/mbs).
} 
offer a security that is backed by an investment asset with a nonrecourse property. In the context of structured finance products, this question is equivalent to discussing why the FI invests in illiquid long-term assets, borrows using nonrecourse short-term securities financed by off-balance-sheet vehicles, and accepts the possibility of providing liquidity support for these vehicles afterward, despite the nonrecourse property. Indeed, if the FI provides liquidity support to off-balance-sheet vehicles, why would the FI use nonrecourse financing of securitization in the first place? Many reasons come to mind, including regulatory and ratings arbitrage, where the FI takes most of the risky loans off its balance sheet to avoid holding costly capital against them, and a low and predictable short-term interest rate environment, where many profitable managers are 'searching for yield'. However, it is interesting to investigate whether the FI should exploit the nonrecourse financing of securitization, even though these institutional features disappear.

To attain this goal, we construct a model in which an FI has investment opportunities but does not have sufficient funds because it must meet other 'liquidity needs' or constraints. Even in this situation, the FI may still invest its own funds and finance the outstanding required funds from external initial uninformed investors (denoted by UIs). To do so, the FI may offer a security that is backed by only the underlying investment asset. For practical or theoretical reasons, we assume that the FI finances the outstanding required funds with short-term securities, thereby allowing the withdrawal of UIs before the cash flows of the asset are realized (in other words, a maturity mismatch exists between the underlying asset and its funding source). ${ }^{3}$ The FI is then exposed to the risk of early withdrawal by the UIs even though the security has the nonrecourse property. However, the FI may have an advantage if the FI acquires information as a delegated monitor on behalf of the UIs when new information is realized after the investment.

The FI then faces two problems. The first is that despite the FI's willingness to continue with the investment, the UIs may withdraw their funds upon the arrival of bad news because they do not have access to the private information held by the FI. If the FI wishes to continue, it must then finance the withdrawn funds from the ex post capital market by issuing claims

\footnotetext{
${ }^{3}$ For simplicity, as in the recent financial crisis literature (see Diamond and Rajan (2009), He and Xiong (2009), Shleifer and Vishny (2010), Uhlig (2010), Huang and Ratnovski (2011), and Acharya and Viswanathan (2011)), we have not modeled the choice of maturity structure specifically until Section 6.1. In Section 6.1, we relax this assumption and show that all of our results still hold. For theoretical studies exploring the reasons different maturities are used, see Calomiris and Kahn (1991), Flannery (1994), Berglof and von Thadden (1994), Diamond and Rajan (2001), Stein (2005), Brunnermeier and Oehmke (2010), and Segura and Suarez (2011).
} 
backed by its own assets and providing liquidity support even though the initial security has the nonrecourse property. ${ }^{4}$ Thus, UIs may create and amplify risk for the FI in the event of their withdrawal, precipitated by adverse perceptions of the investment asset. The second problem is that the private information held by the FI also causes an adverse selection problem in the ex post capital market. This forces the FI to issue an underpriced security. Faced with this combination of problems, the FI must decide whether to issue an initial security with a nonrecourse property in the first place. The FI may also decide whether to undertake information acquisition. In turn, the FI's decisions may cause a problem with overinvestment.

Let us suppose that the FI, the UIs, and the investment assets are identified as the sponsoring bank, ABCP investors, and MBSs and CDOs or their original assets, and that the initial nonrecourse security that allows the early withdrawal of the UIs is interpreted as the ABCP issued by the ABCP conduit or SIV. This model serves to clarify why the FI employs nonrecourse securitized bank loans even though these loans result in losses from suboptimal risk sharing and the FI takes the opportunity to provide liquidity support afterward. The model also sheds light on the question of whether the nonrecourse financing of securitization forces the financial system to perform less efficiently in handling troubled loans, thereby causing an overinvestment problem.

If the FI can choose whether to undertake information acquisition, the main theoretical results of the paper are as follows.

(i) A security with a nonrecourse property backed by a particular asset held by the FI is ex ante preferred to any security backed by its total assets, such as deposit claims.

(ii) Under the optimal nonrecourse security, the UI always withdraws early when future economic conditions are expected to be bad.

(iii) Uninformed finance, inefficient liquidity support, and overinvestment are more likely to arise, despite the nonrecourse property of the security, as the profitability of the asset at the high revenue state is larger or the liquidation value of the asset relative to the amount funded by the UI is smaller or the likelihood of success for the asset investment is expected to be higher.

\footnotetext{
${ }^{4}$ Many recent papers (for example, see Diamond and Rajan (2009), Bolton, Santos, and Scheinkman (2011), and Acharya and Viswanathan (2011)) have tried to explain the fire sale or market freeze phenomena. These models all rely on an implicit assumption that financial intermediaries are constrained from raising outside funds during financial distress. Our paper complements these studies because many financial intermediaries were able to raise outside funds during the recent credit crises.
} 
Our model suggests that it is optimal for the FI to use the nonrecourse financing of securitization initially even though the FI takes the opportunity to provide liquidity support afterward. However, our model also predicts that the nonrecourse financing of securitization, together with short-term maturity financing, is more likely to force the financial system to perform inefficiently in handling troubled loans, thereby causing problems with inefficient liquidity support and overinvestment, if the profitability of the asset at the high revenue state increases or if the liquidation value of the asset relative to the amount funded by the UI decreases or if the likelihood of success with the asset investment increases.

Intuitively, the reason a security with a nonrecourse property backed by a particular asset held by the FI is ex ante preferred to any security backed by its total assets is because the former mitigates the unfavorable effect of the adverse selection problem of the FI raising additional funds to avoid the liquidation of the remaining assets of the FI when the UI withdraws early at the rollover date. More specifically, the nonrecourse security makes its original asset remote from the remaining assets of the FI, thereby enabling the FI to do without the liquidation of the remaining assets of the FI when liquidating the original asset. This makes liquidation of the original asset of the nonrecourse security more attractive for an FI that knows that the original asset is bad. Thus, by establishing bankruptcy remoteness, the nonrecourse security can reduce any additional adverse selection cost in the ex post capital market even though the FI may raise additional funds to provide liquidity support. Such optimality of the nonrecourse security depends on the short-term maturity structure of the security, under which the UI can withdraw early upon the arrival of bad news. Shortterm financing then makes the recourse financing disadvantageous by creating an unfavorable effect of the additional adverse selection problem caused by the early withdrawal of the UI, while making nonrecourse financing advantageous by ruling out the unfavorable effect of raising the FI's borrowing cost in response to the rise in bankruptcy possibility at time 2 .

On the other hand, the early withdrawal of the UI when future economic conditions are expected to be bad stems from the following. If the UI withdraws early, the FI incurs the adverse selection cost of raising additional funds when continuing to invest. By contrast, if the UI continues with the investment, the FI incurs an opportunistic strategy cost because the FI cannot be committed to liquidating a devalued asset. Indeed, the FI prefers to issue a security that induces the early withdrawal of the UI because the adverse selection cost is smaller than the opportunistic strategy cost if the FI can choose whether to undertake information acquisition. 
Given the early withdrawal of the UI when future economic conditions are expected to be bad, the third main result is obtained because, under the conditions stated in this result, an FI with a high-quality asset is less likely to be distinguished from an FI with a devalued asset, even by adjusting the repayments to outside investors in the ex post capital market. A pooling strategy with overinvestment then prevails, because an FI with a devalued asset incurs no additional costs other than repayment when imitating an FI with a high-quality asset. It is not surprising that uninformed finance is more likely to prevail under the conditions stated in the third main result, because information acquired by the FI is less likely to be used.

The logic of this paper, that the nonrecourse financing of securitization is optimal, is closely related to that of Inderst and Mueller (2010), who examine optimal CEO compensation and retirement policy when the CEO is informed privately about the firm's continuation value under his leadership. Inderst and Mueller show that under certain circumstances, steep incentive and severance pay makes quitting attractive for "bad" CEOs who know that they do not have the necessary ability to manage the firm. On the other hand, because the nonrecourse security in our model makes the original asset remote from the remaining assets of the FI, it enables the FI to avoid the liquidation of the remaining assets of the FI when liquidating the original asset. This makes liquidation of the original asset more attractive for an FI that knows that the original asset is bad. If abandoning the investment is viewed as abandoning the firm's management position, the role of nonrecourse financing of securitization in our model seems to correspond well to that of steep incentive and severance pay in Inderst and Mueller.

Holmström and Tirole (1998) investigate a model where firms can meet their future liquidity needs by obtaining an irrevocable line of credit from a financial institution that issues shares backed by claims on its financial position. Winton (2003) considers how the firm's initial choice between debt and equity finance is determined when the financial institution must always meet its interim liquidity needs by issuing securities backed only by its claims on the firm. However, neither of these studies deals with the optimality of the nonrecourse financing of securitization in the ex ante stage, when the FI may provide liquidity support in the ex post stage, despite the nonrecourse property.

This paper relates to the literature on security design and securitization. DeMarzo and Duffie (1999) and DeMarzo (2005) suggest that the process of pooling and tranching in securitization alleviates the underpricing involved in the asset sale because it enhances risk 
diversification and prevents informed intermediates from exploiting uninformed investors under an ex post signaling model. Conversely, by endogenizing both the withdrawal and liquidity support decisions, our model suggests that the nonrecourse property of securitization mitigates the underpricing involved in the additional financing required by the FI when UIs withdraw. Taking the short-term maturity structure as given, Shleifer and Vishny (2010), Uhlig (2010), and Huang and Ratnovski (2011) provide an interesting model of financial intermediaries that securitize, distribute, and trade loans. On the other hand, Brunnermeier and Oehmke (2010) and Segura and Suarez (2011) develop a model of endogenous maturity structure for financial institutions. However, these studies do not explore the optimality of nonrecourse financing with credit enhancement policy in securitization. The difference between our work and the above-mentioned papers is that we derive the interrelated role of the nonrecourse and short-term maturity financing of securitization endogenously. ${ }^{5}$

Indeed, this is the first paper to explore the optimality of nonrecourse financing of securitization when creditors refuse to rollover their lending and withdraw early at the rollover date. Previous papers, such as Benveniste and Berger (1987) and Carlstrom and Samolyk (1995), claim that either exogenous recourse or capital constraints on the sponsoring bank is a key factor that creates the need for securitized bank loans. By contrast, our model attempts to explain endogenously why the nonrecourse financing of securitization occurs even though the FI may provide liquidity support afterward. Ayotte and Gaon (2011) investigate securitization by focusing on the special protection provided by "bankruptcy remoteness" and suggest that the nonrecourse security protects the security holders from dilution of their claims when the debtor-in-possession (DIP) lenders can issue additional senior claims in bankruptcy. However, Ayotte and Gaon do not allow the security holders to withdraw early, nor do they discuss the adverse selection issues. Our paper highlights the role of the nonrecourse security that alleviates the adverse selection incentive in the additional financing of the FI when the security holders withdraw early. ${ }^{6}$

\footnotetext{
${ }^{5}$ Gorton and Souleles (2006) also provide a model for securitization and special-purpose vehicles (SPVs) and show that the repeated relational contract relation explains sponsoring banks' liquidity support of their SPVs in certain states of the world. The relational contract relation has also been explained by Boot, Greenbaum, and Thakor (1993). In our paper, the liquidity support by sponsoring banks can be derived without any reputational considerations. Campello and de Matta (2011) discuss the "empty creditor" problem in credit default swaps (CDSs). Although CDS contracts affect lenders' preference between outof-court restructuring and bankruptcy, Campello and de Matta do not consider the additional financing required by the borrower when the borrower's revenue is short, nor do they discuss the adverse selection issues.

${ }^{6}$ For the literature of nonrecourse financing other than securitization, John (1993) develops an analysis
} 
The paper is organized as follows. Section 2 presents the basic model. Section 3 examines equilibrium under informed finance. Section 4 specifies the uninformed finance equilibrium and discusses the equilibrium configuration of informed and uninformed finance. Further, this section makes it clear that nonrecourse financing of securitization is optimal. Section 5 explores the empirical implications. Section 6 assesses the robustness of the main results. Section 7 concludes the paper. All proofs are in the Appendix.

\section{The Model}

The model has three periods $(t=0,1,2)$. All agents are risk neutral and the risk-free discount rate of interest is zero.

At the beginning of time 0, a financial institution (denoted by FI) holds cash $\bar{I}-I(>0)$ and an asset $A_{O}$ that yields a cash flow at time $2 .{ }^{7}$ In addition, the FI has a new investment $A_{I}$ that yields a cash flow at time 2 but requires a fixed investment outlay of $\bar{I}$ at the beginning of time 0 . Thus, the FI must raise the shortfall of $I$. To finance the amount $I$, the FI issues asset-backed securities that are sold to outside initial investors at the beginning of time 0 . However, the outside initial investors can demand the redemption of these securities at time 1 (for the relaxation of this assumption, see Section 6.1). For brevity, we refer to these outside initial investors as UIs. Further, there are many other outside investors in the ex post capital market. We provide additional details about the financing problem later in this section.

The cash flows of $A_{I}$ and $A_{O}$ for each contingency and their conditional probabilities are given in Table 1. More specifically, at the end of time 0, we allow for two states of nature, $s \in\{g, b\}$. The good state of nature, $g$, occurs with probability $p(\in(0,1))$, and the bad state, $b$, occurs with probability $1-p$. There are three qualities of $A_{I}, q \in\{h, m, \ell\}$, which

of spin-offs that enjoy the benefit of debt-related tax shields. She indicates that the investment disincentives caused by the introduction of risky debt may be reduced by spin-offs. Chemmanur and John (1996) analyze limited-recourse project financing and spin-offs under a symmetric information model driven by considerations of corporate control. Kahn and Winton (2004) suggest that "bipartite" structures - two separate subsidiaries that make loans of similar type but differing risk; for example, a "good bank/bad bank" structure - reduce risk-shifting incentives in the safer subsidiary by insulating safer loans from riskier loans. However, none of these papers discusses the continuation/liquidation decision of the financial intermediary after the withdrawal of uninformed investors under the adverse selection environment. Thus, these papers cannot show clearly that the nonrecourse property of securitization mitigates the underpricing involved in the additional financing of financial intermediation after the uninformed investors withdraw.

${ }^{7}$ Although we may interpret the FI as a nonfinancial firm, our model is particularly appropriate to financial firms because they tend to use higher leverage and more short-term debt. 
differ in their earnings ability. If $s=g$, then $q=h$ occurs with certainty. Alternatively, if $s$ $=b$, then $q=m$ occurs with probability $\delta(\in(0,1))$ and $q=\ell$ occurs with probability $1-$ $\delta$. This framework can capture the situation where the quality of $A_{I}$ deteriorates during the lending period, even though it appears to be good at the time of the initial investment.

If investment in $A_{I}$ is continued at time $2, A_{I}$ generates a cash flow that depends on $q$. If $q=h$, then $A_{I}$ yields a cash flow $R$ with certainty. Next, if $q=m$, then $A_{I}$ yields $R$ with probability $\sigma_{m}(\in(0,1))$ and yields no cash flow with probability $1-\sigma_{m}$. If $q=\ell$, then $A_{I}$ yields $R$ with probability $\sigma_{\ell}(\in(0,1))$ and yields no cash flow with probability $1-\sigma_{\ell}$. As $q$ $=m$ is a higher-quality type than $q=\ell$, we assume that $\sigma_{m}>\sigma_{\ell}$.

For asset $A_{O}$, we assume that because of market- or industry-level fluctuations, the cash flow distributions of $A_{I}$ and $A_{O}$ are correlated: $A_{O}$ yields a cash flow $Z$ with certainty if $A_{I}$ yields $R$, whereas $A_{O}$ yields $Z$ with probability $\sigma_{Z}(\in(0,1))$ and no cash flow with probability $1-\sigma_{Z}$ if $A_{I}$ yields no cash flow. Hence, $\sigma_{Z}$ is interpreted as the probability of success in $A_{O}$ when $A_{I}$ yields no cash flow.

If $A_{I}$ is liquidated at time 1 , it can be redeployed for the value $L$. However, if investment in $A_{I}$ is continued through time 2 , the liquidation value is zero. Conversely, we assume that $A_{O}$ generates no liquidation value at times 1 and 2 . No liquidation value of $A_{O}$ at time 1 is assumed because we focus on the case where the liquidity provision for the FI is limited at time 1 when $s=b$. In fact, our main results hold if the expected cash flow of $A_{O}$ at $s=b$ is sufficiently larger than its liquidation value.

For the information structure, at the end of time 0, each agent can observe the state of nature, $s$, that captures the future economic environment that affects the quality of $A_{I}$. However, as the state of nature reflects immeasurable perceptions in the marketplace about the future economic environment, we assume that it is observable but not verifiable. In addition, at the end of time 0 , the FI can privately observe the quality of $A_{I} \cdot{ }^{8}$ These assumptions imply that only the FI can distinguish between $q=m$ and $q=\ell$, although $q=h$ is observable (but not verifiable). We also assume that both the cash flow and the liquidation value outcomes of $A_{I}$ and $A_{O}$ are observable and verifiable. Finally, we assume that all of the probabilities are common knowledge.

Note that the likelihood of $A_{I}$ or $A_{O}$ generating a positive cash flow is larger for $q=m$ than for $q=\ell$ as long as $\sigma_{m}>\sigma_{\ell}$. Hence, the UI and outside investors in the ex post capital market face adverse selection problems at $s=b$ regardless of whether the FI issues a security

\footnotetext{
${ }^{8}$ The case of an uninformed FI is discussed in Section 4.1.
} 
backed by the cash flows of $A_{I}$, or $A_{O}$, or both.

The structure of the game is illustrated in Figure 1, and is described as follows.

(1) At the beginning of time 0 , the FI places its funds $\bar{I}-I$ in $A_{I}$ and offers the UI an initial security to seek $I$. The UI then decides whether to place funds $I$ in the security.

(2) At the end of time 0 , the state $s$ and the quality $q$ are realized.

(3) The UI decides whether to continue or withdraw at time 1, given its updated beliefs about $q .^{9}$

(a) If the UI chooses to continue, the FI decides whether to continue or liquidate $A_{I}$ at time 1 , given the realized value $q .{ }^{10}$

(b) If the UI withdraws at time 1 , the FI decides whether to continue or liquidate $A_{I}$ at time 1, given the realized value $q$. If the FI chooses to continue, it issues a new security to outside investors in the capital market at time 1 . The outside investors then decide whether to place their funds in the security, given their updated beliefs.

(4) Whenever possible, the beliefs of each agent must satisfy Bayes' rule.

To solve the model, we use the concept of a perfect Bayesian equilibrium.

We are now in a position to consider how the FI finances the amount $I$. In the subsequent analysis, we focus on the effect of the securitization of the cash flow of $A_{I}$ or the cash flows of $A_{I}$ and $A_{O}$. To this end, we formalize the following arrangement. First, we assume that the FI has a superior understanding of the quality of the assets and their likelihood of earning returns, whereas other investors do not. ${ }^{11}$ Hence, if the FI directly sells $A_{I}$ and $A_{O}$, their values are substantially discounted. Second, we assume that the FI cannot raise $I$ by securitizing the cash flow of $A_{O}$ alone until time 1 , because these proceeds have been used for other urgent or opaque liquidity needs of the FI until time 1, when these liquidity needs disappear. Other liquidity needs may include limited-time investment opportunities, the contractual takedown or withdrawal of funds by another customer, or a temporary financial crisis. $^{12}$

\footnotetext{
${ }^{9}$ Allowing the UI to withdraw some but not all of its investment is not optimal because of the linearity of the model.

${ }^{10}$ We assume that the FI retains control over the liquidation decision. This is not restrictive, because the UI is not informed of $q$ at $s=b$, so that under Assumption 2, imposed below, it would always be forced to continue to invest if the UI retained control over the liquidation decision. This assumption is also consistent with actual practice and is supported theoretically by Riddiough (1997), who shows that the junior security holder should control the liquidation decision under asset-backed securities.

${ }^{11}$ This holds not only for many relationship-lending assets but also for MBSs. See Glaeser and Kallal (1997) and DeMarzo (2005).

12 There may be several other reasons the FI is initially willing to invest only a limited amount. For
} 
Hence, at the beginning of time 0 , we focus on the case where the FI issues an initial security backed by the cash flow of $A_{I}$ to raise $I$. Later, we consider an initial security backed by the cash flows of $A_{I}$ and $A_{O}$ to relax the nonrecourse property, and we show that it is still optimal for the FI to issue an initial security backed by the cash flow of $A_{I}$ alone.

As $s$ is unverifiable and $q$ is privately observed at $s=b$ by the FI, the initial security backed by the cash flow of $A_{I}$ is of the debt type, so that the UI agrees to lend $I$ at time 0 and requires a repayment $X$ at time 2 if $A_{I}$ yields $R$, and 0 if $A_{I}$ yields no cash flow. ${ }^{13}$ Because the claims of the UI are secured solely by the cash flow of $A_{I}$, the UI cannot receive anything at time 2 when $A_{I}$ yields no cash flow. If liquidation occurs, the UI secures the liquidation value of the asset in lieu of security repayment. We assume that the claims of the UI take priority over those of the FI. Thus, the receipts from $A_{I}$ are used to repay the UI before any revenues are directed to the FI. The priority structure is justified later.

In fact, the UI keeps the option to withdraw funds at time 1. This is equivalent to assuming that the FI finances the long-term asset $A_{I}$ by issuing a short-term security at time 0 and letting the UI roll it over at time 1. This assumption seems to correspond well to the nature of short-term rollover debt such as commercial paper or margins and collateral requirements in financial contracts. ${ }^{14}$ However, in Section 6.1, even though the FI can choose the maturity structure of debt, we show that our main results are unaffected.

Thus, the UI can choose whether to withdraw its funds at time 1, given its updated beliefs about $q$ and the security provisions, because the UI observes $s$ at the end of time 0. First, suppose that the UI does not withdraw. The FI must now decide whether to continue or liquidate. If the FI chooses to continue, investment in $A_{I}$ is continued through time 2 . If the FI chooses to liquidate, each agent receives its claim according to its priority structure at time 1. Next, suppose that the UI withdraws. Again, the FI must decide whether to continue or liquidate. However, if the FI chooses to continue, it must assure the UI of $I$ at time $1 .{ }^{15}$

instance, the FI may be wealth constrained or may prefer to have only limited exposure to an asset for reasons of risk shifting or capital constraint (see Jones, 2000).

${ }^{13}$ It is not useful for the FI to report the realization of $q$ to the UI before the UI makes its withdrawal decision. This is because the FI always reports $q=m$ because $q$ is private information at $s=b$ and the FI incurs no cost by reporting $q=m$.

${ }^{14}$ Short-term maturity financing is assumed by most recent studies of securitization. See the literature in footnote 3 .

${ }^{15}$ If the FI can choose a smaller promised payment than $I$ when the UI withdraws, the FI is more likely to continue to invest in $A_{I}$. However, this implies that inefficient continuation is more likely to occur, because inefficiency arises only as inefficient continuation in the present model. By contrast, suppose that the FI can choose a larger promised payment than $I$ - that is, $I^{\prime}>I$ - when the UI withdraws. Regardless of whether the equilibrium is located in the separating or the pooling region when the FI's promised payment is fixed 
Thus, the FI must finance the funds withdrawn by the UI, $I$, at time 1 by raising new funds from outside investors in the capital market. This can be viewed as the FI giving liquidity support to the deteriorated asset, although the FI uses the nonrecourse security. Indeed, after withdrawal, the UI may be allowed to refinance the new funds for the FI in the capital market, or it may not do so because of an internal code for risky investment. Throughout most of this paper, we consider the latter. We discuss the former in the Appendix, and show that our main results are unaffected qualitatively by the modification. If the FI liquidates, each agent receives its claim according to its priority structure at time 1.

If the UI withdraws at time 1 but the FI wishes to continue, we assume that the FI must issue a new security backed by $A_{I}$ and $A_{O}$ in the capital market at time 1 . The optimality of this form of security is proved in Section 6.2. As $q$ is privately observed by the FI at $s$ $=b$, and as the liquidation values of both $A_{I}$ and $A_{O}$ are zero at time 2 , the new security depends only on the cash flows generated by $A_{I}$ and $A_{O}$. This implies that in exchange for funding $I$, outside investors require a repayment $Y_{R+Z}$ at time 2 if $A_{I}$ and $A_{O}$ yield a cash flow $R+Z$, a repayment $Y_{Z}$ if $A_{I}$ and $A_{O}$ yield $Z$, and 0 if $A_{I}$ and $A_{O}$ yield no cash flow. We also assume the monotonicity condition $Y_{R+Z} \geq Y_{Z}$. This is standard in the security design literature and can be formally justified on the grounds of moral hazard. ${ }^{16}$

Several remarks on the initial security issued at time 0 are in order. First, we rule out the possibility that the FI renegotiates the security provision with the UI after the realization of $s$ and $q$ but before the continuation/withdrawal decision of the UI. This assumption is plausible because we interpret our model in the securitization setting, where the UI comprises numerous unknown security holders.

Second, the priority of the claims of the UI in liquidation can be justified in several ways. For one thing, Axelson, Stromberg, and Weisbach (2009) assume that the initial adverse selection problem of FIs causes strategic defaults of institutions that are not serious. In our context, this implies that there are many fly-by-night operators that the UI cannot

at $I$ after the withdrawal of the UI, the FI with $q=m$ has no incentive to pay $I^{\prime}$ after the withdrawal of the UI. Indeed, in the separating region, the additional amount $I^{\prime}-I$ merely causes the FI with $q=m$ to incur more costs and cannot serve to mitigate adverse selection incentives. In the pooling region, the repayment level $Y^{m}$ at time 2 that can deter the FI with $q=\ell$ from mimicking the FI with $q=m$ does not depend on $I^{\prime}$ because the self-selection constraint does not depend on $I^{\prime}$. Only the participation constraint of outside investors in the capital market at $t=1$ is affected by $I^{\prime}$. Hence, again, $I^{\prime}-I$ merely increases the costs incurred by the FI with $q=m$.

${ }^{16}$ For justification, see Innes (1990), Nachman and Noe (1994), Axelson (2007) and Axelson, Stromberg and Weisbach (2009). Because Winton (2003) assumes that the institution meets any liquidity needs by issuing equity or debt claims on its position in the firm, his model also assumes monotonicity. 
distinguish from a serious FI. We may assume that if the FI can capture some part of the liquidation value, there are many fly-by-night operators who can earn positive rents by raising $I$, investing $I$ in assets with a maximum payoff less than $I$ (but with a positive liquidation value) and then liquidating the assets. In this situation, the UI cannot break even. Thus, any optimal security must require that the claims of the FI are junior to the claims of the UI. It is also possible to justify the priority rule by the unmodeled moral hazard and information problems of the FI. Winton (1995) argues that most junior claimants should monitor the project. If the FI does not monitor the asset $A_{I}$ when it has senior claims, our priority rule may be justified, because the cash flow revenues drop and the security is substantially underpriced unless the FI has junior claims. ${ }^{17}$ This priority structure is commonly observed in practice, where the FI provides credit enhancement such as senior/subordinate structures, particularly in asset-backed securitization. Bolton and Freixas (2000) assume the priority structure observed in practice and show that it is the main source (in their model) behind the banks' incentive to securitize; that is, banks can do better by undoing priority ordering through securitization.

To focus on the issue of withdrawal of the UI and to evaluate economic efficiency, we impose the following parametric restrictions on $A_{I}$.

Assumption 1: $\sigma_{\ell} R<L<I<\sigma_{m} R$.

Assumption 2: $L<\sigma_{b} R$, where $\sigma_{b} \equiv \delta \sigma_{m}+(1-\delta) \sigma_{\ell}$.

Assumption 3: The FI always has an incentive to invest in $A_{I}$ at time 0 because $p$ is sufficiently large or $\bar{I}$ is not sufficiently large.

Assumption 1 implies that it is ex post efficient to continue to invest in $A_{I}$ (liquidate $A_{I}$ ) when $q=m(q=\ell)$. This is because if the FI continues when $q=m(q=\ell)$, the expected cash flow of $A_{I}, \sigma_{m} R\left(\sigma_{\ell} R\right)$, is larger (smaller) than the liquidation value of $A_{I}$, L. Given that $\sigma_{b}$ is the probability of $A_{I}$ yielding $R$ conditional on $s=b$, Assumption 2 indicates that it is ex post efficient to continue to invest in $A_{I}$ if the quality of $A_{I}$ is uncertain when $s=$

\footnotetext{
${ }^{17}$ In addition, if $A_{I}$ is liquidated at $s=b$, the UI may face liquidity needs because of the withdrawal of other investors from its own funds, unless its claims in liquidation are large. The UI is then forced to liquidate its own assets if it cannot raise the liquidity needs. This possibility is most likely to occur if the UI is a money market mutual fund, which can neither expect a new inflow of funds in this situation nor borrow to continue to invest. Even if the UI can raise the required funds, it may be forced to promise repayment in excess of the fair risk-adjusted level. This is because the adverse selection or moral hazard problems not modeled here undermine its credibility. This tendency is aggravated if the situation of distress causes dislocation in the credit and funding markets that could restrict the overall provision and channeling of credit, or if it induces rating agencies to subsequently downgrade securities issued by the UI.
} 
b. In our model, if it is optimal to invest in $A_{I}$ at time 0 , social welfare depends only on the decision of whether to continue to invest in $A_{I}$ or liquidate $A_{I}$ at time 1 when $s=b$. Assumptions 1 and 3 thus ensure that continuing to invest in $A_{I}$ (liquidating $A_{I}$ ) for $q=m$ $(q=\ell)$ is the first-best allocation.

Before proceeding to characterize the equilibrium, we put two additional assumptions. First, the following assumption serves to simplify the analysis of the ex post capital market. Assumption 4: $Z \geq \max \left[\frac{\sigma_{\ell} R}{\sigma_{\ell}+\left(1-\sigma_{\ell}\right) \sigma_{Z}}, \frac{I}{\sigma_{\ell}+\left(1-\sigma_{\ell}\right) \sigma_{Z}}\right]$.

Assumption 4 implies that if $q=\ell$, the expected cash flow of $A_{O},\left[\sigma_{\ell}+\left(1-\sigma_{\ell}\right) \sigma_{Z}\right] Z$, is larger than or equal to that of $A_{I}, \sigma_{\ell} R$, and is also larger than or equal to the investment of the UI, $I$. This ensures, in a simplified way, that the FI has an incentive to raise the withdrawn funds by securitizing $A_{I}$ and $A_{O}$ (or $A_{O}$ alone) at time 1 when $s=b$. In the Appendix, we show that our main results are unaffected qualitatively if this assumption is relaxed.

Second, we impose the following assumption to simplify the subsequent analysis.

Assumption 5: (i) $\delta>1-\frac{\sigma_{m}}{\sigma_{m}-\sigma_{\ell}} \frac{p\left(1-\sigma_{b}\right)}{p+(1-p) \sigma_{b}}$, and (ii) $\delta>\frac{\left(1-\sigma_{Z}\right) \sigma_{\ell}}{\sigma_{Z}+\left(1-\sigma_{Z}\right) \sigma_{\ell}}$.

Assumption $5(\mathrm{i})$ is rewritten as an assumption with respect to $p$ : (i') $p>\frac{(1-\delta)\left(\sigma_{m}-\sigma_{\ell}\right)}{1-\sigma_{b}}$. $^{18}$ Assumption 5(i) and (ii) mainly serves to simplify the equilibrium configuration. Assumption $5(\mathrm{i})$ is also used to ensure that the FI's claim in the optimal initial security is nonnegative. In the Appendix, we discuss the relaxation of Assumption 5.

Assumptions 1-5 can be satisfied when $\delta$ (the probability of $q=m$ conditional on $s=b$ ) is large, when $p$ (the probability of $s=g$ or $q=h$ ) is large, when $Z$ (the cash flow of $A_{O}$ ) is large, when $\sigma_{Z}$ (the probability of success in $A_{O}$ if $A_{I}$ generates no cash flow) is large, and when $\bar{I}$ (the fixed investment outlay of $A_{I}$ ) is small.

\section{Equilibrium}

The game is solved through backward induction. In Section 3.1, we first examine the continuation/liquidation decision of the FI at time 1, taking as given the continuation/withdrawal decision of the UI at time 1 . If the FI chooses to continue to invest in $A_{I}$ and raises new funds after the withdrawal of the UI, we also investigate the new security offer decision of the FI at time 1 in the ex post capital market. Taking into account the optimal decisions of the FI

\footnotetext{
${ }^{18}$ Note that $\frac{(1-\delta)\left(\sigma_{m}-\sigma_{\ell}\right)}{1-\sigma_{b}}<1$ and $\partial\left(\frac{(1-\delta)\left(\sigma_{m}-\sigma_{\ell}\right)}{1-\sigma_{b}}\right) / \partial \delta<0$.
} 
at time 1, we next discuss the continuation/withdrawal decision of the UI at time 1. Indeed, this UI's decision depends on the initial security offer at time 0. Thus, like a Stackelberg leader, the FI can affect the continuation/withdrawal decision of the UI by adjusting the initial security offer. In Section 3.2, taking account of the optimal decisions of the FI and the UI at time 1, we characterize the optimal initial security offer of the FI at time 0.

\subsection{The optimal decisions of the FI and the UI at time 1.-}

We must first consider the optimal decisions of the FI and the UI at $s=g$. Because $A_{I}$ then yields $R$ with certainty, whereas the junior position of the FI's claim implies that the FI receives nothing if it liquidates $A_{I}$, it is trivial that the FI chooses to continue, regardless of whether the UI withdraws or continues. Given this, the UI's ex ante individual rationality constraint ensures that the UI chooses to continue.

However, if $s=b$, the cash flow of $A_{I}$ depends on $q$, and is not necessarily positive. Because only the FI privately knows $q$ at time 1, the analysis is much more complicated. In the rest of this subsection, we focus on the case of $s=b$.

In Section 3.1.1, we examine the continuation/liquidation decision of the FI after the UI continues at $s=b$. In Section 3.1.2, we discuss the continuation/liquidation decision of the FI after the UI withdraws at $s=b$, and derive the optimal new security offer of the FI in the ex post capital market when the FI continues to invest in $A_{I}$ and raises new funds after the withdrawal of the UI. As the FI privately knows $q$, the equilibrium in the ex post capital market is a signaling equilibrium, which is featured in Lemma 2. Taking account of the optimal decisions of the FI at time 1 derived in Sections 3.1.1 and 3.1.2, we clarify the continuation/withdrawal decision of the UI at $s=b$ at time 1 in Section 3.1.3.

\subsubsection{The continuation/liquidation decision of the FI after the UI continues If} the UI continues, irrespective of whether the FI has $q=m$ or $q=\ell$, the FI also continues because it receives nothing in liquidation. As a result, we present the following lemma.

Lemma 1: When $s=b$, the FI always continues to invest in $A_{I}$ after the UI continues to invest in $A_{I}$ at time 1 .

Lemma 1 suggests that if the UI does not withdraw at $s=b$, the FI with $q=\ell$ cannot commit to liquidating $A_{I}$; thus, overinvestment occurs for the bad earning ability of the $A_{I}$ $(q=\ell)$. 


\subsubsection{The continuation/liquidation decision of the FI after the UI withdraws}

In this case, suppose that the FI chooses to liquidate. Then, the junior position of the FI's claim forces the FI to receive nothing. On the other hand, suppose that the FI chooses to continue. Then, the FI must finance $I$ to be repaid to the UI in the capital market. The problem is that the FI knows $q$, while outside investors in the capital market do not at $s$ $=b$. Outside investors can only rationally revise their beliefs after they observe both the realization of $s=b$ and the FI's continuation/liquidation and security design decisions at time 1. As the FI has private information about $q$, we need to consider not only the pooling strategy of the FI that does not depend on $q$ but also the separating strategy of the FI that does depend on $q$. To refine the optimal strategy of the FI that chooses to continue, we use the requirement that the equilibrium satisfies the D1 criterion in Banks and Sobel (1987). ${ }^{19}$ Under D1, outside investors that observe an out-of-equilibrium strategy focus their beliefs on the type of FI that would benefit them the most from choosing this strategy.

To characterize the signaling equilibrium in the ex post capital market, let FI $m$ denote the FI with $q=m$, and let FI $\ell$ denote the FI with $q=\ell$. Under the pooling strategy, we need not differentiate between the FI $m$ and the FI $\ell$. Under the separating strategy, define $\left(Y_{R+Z}^{m}, Y_{Z}^{m}\right)$ as $\left(Y_{R+Z}, Y_{Z}\right)$ issued by the FIm. Define $\Re_{1} \equiv \frac{\sigma_{\ell}+\left(1-\sigma_{\ell}\right) \sigma_{Z}}{\sigma_{\ell}\left[\sigma_{b}+\left(1-\sigma_{b}\right) \sigma_{Z}\right]}$ and $\Re_{2} \equiv \frac{\sigma_{\ell}+\left(1-\sigma_{\ell}\right) \sigma_{Z}}{\sigma_{\ell}\left[\sigma_{m}+\left(1-\sigma_{m}\right) \sigma_{Z}\right]}$, where $\Re_{1}>\Re_{2} \cdot{ }^{20}$ Then, we have:

Lemma 2: Suppose that the UI withdraws when $s=b$.

(i) If $\frac{1}{\sigma_{\ell}}>\frac{R}{I} \geq \Re_{1}$, the pooling strategy prevails: both the FIm and the FIl prefer to continue to invest in $A_{I}$. The repayment level, $Y^{P}$, is $Y^{P}=\frac{I}{\sigma_{b}+\left(1-\sigma_{b}\right) \sigma_{Z}}$.

(ii) If $\Re_{1}>\frac{R}{I} \geq \Re_{2}$, the separating strategy prevails: the FIm continues to invest in $A_{I}$ while the FIl liquidates $A_{I}$. The repayment schedule is given by $Y_{R+Z}^{m}=Y_{Z}^{m}=Y^{m}$, where $Y^{m}=\frac{\sigma_{\ell} R}{\sigma_{\ell}+\left(1-\sigma_{\ell}\right) \sigma_{Z}}\left(>\frac{I}{\sigma_{m}+\left(1-\sigma_{m}\right) \sigma_{Z}}\right.$ in this range of $\left.\frac{R}{I}\right)$.

(iii) If $\Re_{2}>\frac{R}{I}>\frac{1}{\sigma_{m}}$, the separating strategy prevails: the FIm continues to invest in $A_{I}$ while the FIl liquidates $A_{I}$. The repayment schedule is given by $Y_{R+Z}^{m}=Y_{Z}^{m}=Y^{m}$, where $Y^{m}=\frac{I}{\sigma_{m}+\left(1-\sigma_{m}\right) \sigma_{Z}}$.

Lemma 2 suggests that despite the nonrecourse financing, the FI raises new funds and gives inefficient liquidity support in some cases after the withdrawal of the UI. Indeed, if the profitability of $A_{I}$ at success is sufficiently high $\left(\frac{1}{\sigma_{\ell}}>\frac{R}{I} \geq \Re_{1}\right)$, the pooling strategy

\footnotetext{
${ }^{19}$ DeMarzo and Duffie (1999) and Winton (2003) also impose the D1 refinement criterion.

${ }^{20}$ Note that $\frac{R}{I}$ is restricted by the range $\left(\frac{1}{\sigma_{m}}, \frac{1}{\sigma_{\ell}}\right)$ under Assumption 1. It is immediately apparent from $\sigma_{m}>\sigma_{b}>\sigma_{\ell}$ that $\frac{1}{\sigma_{m}}<\Re_{2}<\Re_{1}<\frac{1}{\sigma_{\ell}}$.
} 
dominates the separating strategy and brings about efficient investment for the medium earning ability of $A_{I}(q=m)$, but causes overinvestment (inefficient continuation) for the bad earning ability of $A_{I}(q=\ell)$ after the UI withdraws. Note that $\Re_{1}$ is the threshold of $\frac{R}{I}$ above which both the FI $m$ and the FI $\ell$ prefer to continue under the pooling strategy. By contrast, if the profitability of $A_{I}$ at success is not sufficiently high $\left(\Re_{1}>\frac{R}{I}>\frac{1}{\sigma_{m}}\right)$, the FI raises new funds only if $q=m$. Thus, the separating strategy dominates the pooling strategy and creates efficient investment both for $q=m$ and for $q=\ell$ after the UI withdraws. However, if the profitability of $A_{I}$ at success is moderate $\left(\Re_{1}>\frac{R}{I} \geq \Re_{2}\right)$, the FIm must repay outside investors' premiums in excess of the fair risk-adjusted level because $\frac{\sigma_{\ell} R}{\sigma_{\ell}+\left(1-\sigma_{\ell}\right) \sigma_{Z}}$ $\geq \frac{I}{\sigma_{m}+\left(1-\sigma_{m}\right) \sigma_{Z}}$. Note that $\Re_{2}$ is the threshold of $\frac{R}{I}$ above which the FI $m$ must repay outside investors in excess of the fair risk-adjusted level under the separating strategy.

The intuition is as follows. Under the pooling strategy, if investment in $A_{I}$ is continued, the FI with $q$ receives $R$ with probability $\sigma_{q}$ but repays $Y^{P}=\frac{I}{\sigma_{b}+\left(1-\sigma_{b}\right) \sigma_{Z}}$ with probability $\sigma_{q}$ $+\left(1-\sigma_{q}\right) \sigma_{Z}$. If $A_{I}$ is liquidated, it follows from $L<I$ that the FI cannot receive anything regardless of its $q$. Hence, given the definition of $\Re_{1}$, both the FIm and the FI $\ell$ prefer to continue, raise new funds, and give liquidity support as long as the profitability of $A_{I}$ at success is sufficiently high $\left(\frac{1}{\sigma_{\ell}}>\frac{R}{I} \geq \Re_{1}\right)$. However, for $\Re_{1}>\frac{R}{I}$, the FI $\ell$ has no incentive to continue for the repayment level $Y^{P}$, because its expected payoff at date 1 is negative.

On the other hand, under the separating strategy, the repayment schedule is given by $Y_{R+Z}^{m}=Y_{Z}^{m}=Y^{m}$ to minimize the underpricing of the security, as predicted by the "folklore proposition of debt" in the standard security design literature (Axelson (2007)). If outside investors know the quality of $A_{I}$, only the FIm continues and raises new funds because the FI $\ell$ prefers liquidation under Assumption 1. In fact, outside investors do not know the quality of $A_{I}$. Because the FI receives nothing in liquidation, the FI $\ell$ may have an incentive to mimic the FIm. However, if the profitability of $A_{I}$ at success is sufficiently low $\left(\Re_{2}>\frac{R}{I}>\right.$ $\left.\frac{1}{\sigma_{m}}\right)$, the expected cash flow received by the FI $\ell$ in continuation is smaller than its expected repayment level. Hence, in this case, the FI $\ell$ prefers to liquidate, while the FI $m$ prefers to continue. By contrast, if the profitability of $A_{I}$ at success is not sufficiently low $\left(\frac{1}{\sigma_{\ell}}>\frac{R}{I}\right.$ $\geq \Re_{2}$ ), the FI $\ell$ has an incentive to mimic the FIm if the repayment level demanded of the FI $m, Y^{m}$, is the same as the fair risk-adjusted one, $\frac{I}{\sigma_{m}+\left(1-\sigma_{m}\right) \sigma_{Z}}$. Thus, $Y^{m}$ must be adjusted upward until the FI $\ell$ has no incentive to mimic the FIm. As a result, $Y^{m}$ must be set equal to $\frac{\sigma_{\ell} R}{\sigma_{\ell}+\left(1-\sigma_{\ell}\right) \sigma_{Z}}$; and $\Re_{2}$ turns out to be the threshold defined above.

Because the FIm knows that its quality of $A_{I}$ is $q=m$, it may prefer to separate itself from 
the FI $\ell$. Hence, the FIm may be more willing to make a higher repayment. However, if the $\mathrm{FI} \ell$ imitates the FI $m$ that chooses to continue, the FI $\ell$ incurs only the same repayment to outside investors that the FIm does. Indeed, whether the pooling or the separating strategy is used by the FI depends on the profitability of $A_{I}$ at success, $\frac{R}{I}$.

If the profitability of $A_{I}$ at success is sufficiently high $\left(\frac{1}{\sigma_{\ell}}>\frac{R}{I} \geq \Re_{1}\right)$, the repayment level $Y^{P}$ under the pooling strategy where both the FI $m$ and the FI $\ell$ continue is sufficiently low relative to $R$ that the expected payoff of the FI $\ell$ is nonnegative. As it is very costly for the FI $m$ to prevent the FI $\ell$ from mimicking the FI $m$ 's strategy in this range of $\frac{R}{I}$, this induces the FIm to select the pooling strategy.

By contrast, if the profitability of $A_{I}$ at success is not sufficiently high $\left(\Re_{1}>\frac{R}{I}>\frac{1}{\sigma_{m}}\right)$, $Y^{P}$ is sufficiently high relative to $R$ that the FI $\ell$ has no incentive to continue. This rules out the pooling strategy where both the FI $m$ and the FI $\ell$ continue. Furthermore, the FI $m$ always has the greatest incentive to continue, regardless of the repayment level. Thus, the FI most willing to continue at any repayment level turns out to be the FIm. Under D1 beliefs, if outside investors observe that the FI continues and seeks additional funding with a repayment level that induces the FI $\ell$ to liquidate, they believe that the FI is the FIm. This rules out the pooling strategy where both the FIm and the FI $\ell$ liquidate $A_{I}$. Hence, only the separating strategy survives. In addition, if the profitability of $A_{I}$ at success is sufficiently low $\left(\Re_{2}>\frac{R}{I}>\frac{1}{\sigma_{m}}\right)$, the FI $\ell$ has no incentive to continue for the repayment level $Y^{m}$ that would prevail if outside investors were able to learn the quality of $A_{I}$. Thus, in this range, the FIm need not repay outside investors any premiums in excess of the fair risk-adjusted level.

The difference between the results of Lemma 2 and the standard signaling model is that the pooling strategy prevails if $\frac{R}{I} \geq \Re_{1}$. This is because the FI $\ell$ incurs no additional costs other than repayment when imitating the FIm that chooses to continue. Hence, the FIm cannot prevent the imitating strategy of the FI $\ell$ if $\frac{R}{I} \geq \Re_{1}$.

3.1.3. The continuation/withdrawal decision of the UI If the UI continues, Lemma 1 shows that both the FIm and the FI $\ell$ continue. However, if the UI withdraws, Lemma 2 suggests that we must examine the following three cases: (i) $\frac{1}{\sigma_{\ell}}>\frac{R}{I} \geq \Re_{1}$; (ii) $\Re_{1}>\frac{R}{I} \geq$ $\Re_{2}$; and (iii) $\Re_{2}>\frac{R}{I}>\frac{1}{\sigma_{m}}$. Then, we obtain the following lemma. ${ }^{21}$

Lemma 3: Suppose that $s=b$.

\footnotetext{
${ }^{21}$ We assume that the UI chooses to continue if it is indifferent between continuing and withdrawing.
} 
(i) If $\frac{1}{\sigma_{\ell}}>\frac{R}{I} \geq \Re_{1}$, the UI continues if $\sigma_{b} X \geq I$. Otherwise, the UI withdraws.

(ii) If $\Re_{1}>\frac{R}{I}>\frac{1}{\sigma_{m}}$, the UI continues if $\sigma_{b} X \geq \delta I+(1-\delta) L$. Otherwise, the UI withdraws.

Intuitively, if the UI continues, both the FIm and the FI $\ell$ always continue. As the UI does not know the type of FI at $s=b$, its interim expected payoff is $\sigma_{b} X$. On the other hand, if the UI withdraws, Lemma 2 indicates that the UI expects to receive $I$ if the profitability of $A_{I}$ at success is sufficiently high $\left(\frac{1}{\sigma_{\ell}}>\frac{R}{I} \geq \Re_{1}\right)$, but only $\delta I+(1-\delta) L$ if the profitability of $A_{I}$ at success is not sufficiently high $\left(\Re_{1}>\frac{R}{I}>\frac{1}{\sigma_{m}}\right)$. Thus, comparing the expected payoff of the UI in continuation with that in liquidation, we derive the result of Lemma 3.

\subsection{The optimal initial security offer of the FI at time 0 .-}

The arguments in Section 3.1 suggest that the optimal initial security at time 0 depends on whether the UI continues or withdraws at $s=b$. In addition, as the Stackelberg leader, the FI can induce the UI to continue or withdraw at $s=b$ by adjusting the security provisions.

Now, given Lemmas 1-3, we divide the analysis for the optimal initial security offer into the following three cases: (i) $\frac{1}{\sigma_{\ell}}>\frac{R}{I} \geq \Re_{1}$; (ii) $\Re_{1}>\frac{R}{I} \geq \Re_{2}$; and (iii) $\Re_{2}>\frac{R}{I}>\frac{1}{\sigma_{m}}$. For each of the above three cases, we further need to take account of two situations: (a) where the UI is induced to continue at $s=b$; and (b) where the UI is induced to withdraw at $s=b$. By comparing the ex ante expected payoffs of the FI in the two situations, we can determine the optimal security offer of the FI at time 0 for each of the three cases. In the proof of Lemma 4 in the Appendix, we formalize the maximization problems and derive the optimal security offer in each of the three cases.

Lemma 4 characterizes the optimal initial security offer. The following definitions are useful in stating Lemma $4: \varphi(\mathcal{L}) \equiv \frac{\Re_{1}}{(1-p) \sigma_{b}}\left\{(1-\delta)\left[p+2(1-p) \sigma_{b}\right] \mathcal{L}+\delta\left[p+2(1-p) \sigma_{b}\right]-\right.$ $\left.\sigma_{b}\right\}$ and $\psi(\mathcal{L}) \equiv \max \left(\min \left(\varphi(\mathcal{L}), \Re_{1}\right), \Re_{2}\right),{ }^{22}$ where $\mathcal{L} \equiv \frac{L}{I}$ is the ratio of the liquidation value of $A_{I}$ to the funding amount of the UI.

Lemma 4: (i) If $\frac{1}{\sigma_{\ell}}>\frac{R}{I} \geq \Re_{1}$, the equilibrium (denoted by IFEP) is determined so that the FI offers a security at time 0 that induces the UI to withdraw when $s=b$ at time 1 . The optimal initial security is $X^{*}=I$. After the withdrawal of the UI, both the FIm and the FIl prefer to continue to invest in $A_{I}$. The repayment level from the FI to outside investors in the new security at time $1, Y^{*}$, is $Y^{*}=\frac{I}{\sigma_{b}+\left(1-\sigma_{b}\right) \sigma_{Z}}$.

(ii) If $\Re_{1}>\frac{R}{I} \geq \psi(\mathcal{L})$, the equilibrium (denoted by IFEC) is determined so that the FI offers

\footnotetext{
${ }^{22}$ Note that $\varphi^{\prime}(\mathcal{L})>0$ and $\varphi(1)>\Re_{1}$.
} 
a security at time 0 that induces the UI to continue when $s=b$ at time 1 . The optimal initial security is $X^{*}=\frac{\delta I+(1-\delta) L}{\sigma_{b}}$. Both the FIm and the FIl continue to invest in $A_{I}$ when $s=b$ at time 1 .

(iii) If $\psi(\mathcal{L})>\frac{R}{I}>\frac{1}{\sigma_{m}}$, the equilibrium (denoted by IFES) is determined so that the FI offers a security at time 0 that induces the UI to withdraw when $s=b$ at time 1 . The optimal initial security is $X^{*}=\frac{[1-(1-p) \delta] I-(1-p)(1-\delta) L}{p}$. After the withdrawal of the UI, the FIm continues while the FI liquidates. Furthermore, $Y^{*}=\frac{\sigma_{\ell} R}{\sigma_{\ell}+\left(1-\sigma_{\ell}\right) \sigma_{Z}}$ if $\psi(\mathcal{L})>\frac{R}{I} \geq \Re_{2}$, and $Y^{*}=\frac{I}{\sigma_{m}+\left(1-\sigma_{m}\right) \sigma_{Z}}$ if $\Re_{2}>\frac{R}{I}>\frac{1}{\sigma_{m}}$.

If the profitability of $A_{I}$ at success is sufficiently high $\left(\frac{1}{\sigma_{\ell}}>\frac{R}{I} \geq \Re_{1}\right)$, the optimal initial security induces the UI to withdraw when $s=b$ but causes overinvestment (inefficient continuation) for the poor earning ability of $A_{I}(q=\ell)$ because the FI $\ell$ continues. This implies that despite the nonrecourse security, liquidity support can arise for $q=\ell$ even though the UI withdraws. This also means that the FI $\ell$ can raise sufficient funding for the deteriorated $A_{I}$ in this case. In this sense, capital market pricing does not work well.

If the profitability of $A_{I}$ at success is moderate but is still high enough relative to $\mathcal{L}\left(\Re_{1}\right.$ $>\frac{R}{I} \geq \psi(\mathcal{L})$ ), the optimal initial security induces the UI to continue when $s=b$ and causes overinvestment for $q=\ell$ because the FI $\ell$ continues. Unlike the case of Lemma 4(i), this inefficiency arises when the UI continues at $s=b$. In addition, $\frac{R}{I}=\psi(\mathcal{L})$ is a threshold line above which the FI induces the UI to continue at $s=b$ in the range $\frac{R}{I} \in\left[\Re_{2}, \Re_{1}\right)$.

If the profitability of $A_{I}$ at success is moderate but is not high enough relative to $\mathcal{L}(\psi(\mathcal{L})$ $\left.>\frac{R}{I} \geq \Re_{2}\right)$ or is sufficiently low $\left(\Re_{2}>\frac{R}{I}>\frac{1}{\sigma_{m}}\right)$, the optimal initial security induces the UI to withdraw when $s=b$ and leads to efficient investment for both $q=m$ and $q=$ $\ell$ because the FIm continues while the FI $\ell$ liquidates. Thus, in this case, capital market pricing works well so that the FI raises new funds only if $q=m$. However, the FI $m$ must repay outside investors' premiums if $\psi(\mathcal{L})>\frac{R}{I} \geq \Re_{2}$, whereas the FIm only needs to repay outside investors the fair risk-adjusted level if $\Re_{2}>\frac{R}{I}>\frac{1}{\sigma_{m}}$.

To summarize, the optimal initial security induces the UI to withdraw when $s=b$, except for the case in which the profitability of $A_{I}$ at success is moderate but is still high enough relative to $\mathcal{L}\left(\Re_{1}>\frac{R}{I} \geq \psi(\mathcal{L})\right)$. Hence, the continuation/liquidation decision of the FI is given by Lemma 2 , except for $\frac{R}{I} \in\left[\psi(\mathcal{L}), \Re_{1}\right)$. Despite the nonrecourse financing, if the UI withdraws, the optimal initial security induces the FI $m$ to raise new funds and provide liquidity support at $s=b$, while it induces the FI $\ell$ to raise new funds and give liquidity 
support at $s=b$ only if the profitability of $A_{I}$ at success is sufficiently high $\left(\frac{1}{\sigma_{\ell}}>\frac{R}{I} \geq \Re_{1}\right)$.

The intuition for Lemma 4 is explained as follows. Suppose that the profitability of $A_{I}$ at success is sufficiently high $\left(\frac{1}{\sigma_{\ell}}>\frac{R}{I} \geq \Re_{1}\right)$. Lemmas 1 and $2(\mathrm{i})$ show that regardless of whether the FI induces the UI to continue or withdraw at $s=b$, the FI always continues to invest until time 2. To check whether the FI induces the UI to continue or withdraw at $s=b$, we need to compare the financing costs of these strategies. If the FI induces the UI to continue, the FI must increase the face value of the initial security, $X$, to $\frac{I}{\sigma_{b}}$, which guarantees the UI the same interim expected payoff as that from withdrawal. By contrast, if the FI induces the UI to withdraw, both the FI $m$ and the FI $\ell$ raise additional funds because they prefer to continue to invest in this range of $\frac{R}{I}$. Thus, the UI can receive the certain redemption amount of $I$ regardless of $q=m$ or $q=\ell$. However, this causes a severe adverse selection problem in the ex post capital market. Hence, the pooling equilibrium prevails so that both the FIm and the FI $\ell$ finance $I$ at time 1 by issuing the same new security with $Y^{*}=\frac{I}{\sigma_{b}+\left(1-\sigma_{b}\right) \sigma_{Z}}($ see Lemma 2(i)). Indeed, the former cost of the FI inducing the UI to continue outweighs the latter cost of the FI inducing the UI to withdraw, because the FI must pay $\frac{I}{\sigma_{b}}$ even at $s=g$ in the former case. Hence, the FI prefers to induce the UI to withdraw at $s=b$. Then, the FI can always raise funds and give liquidity support at $s=b$ so that overinvestment arises for $q=\ell$.

Next, suppose that the profitability of $A_{I}$ at success is moderate $\left(\Re_{1}>\frac{R}{I} \geq \Re_{2}\right)$. If the FI induces the UI to continue at $s=b$, Lemma 1 implies that overinvestment occurs for $q=\ell$. On the other hand, if the FI induces the UI to withdraw at $s=b$, Lemma 2(ii) indicates that the FI $m$ continues while the FI $\ell$ liquidates. Hence, efficient investment is attained. However, the FIm must repay outside investors' premiums in excess of the fair risk-adjusted level to finance the withdrawn funds. To check which strategy the FI chooses, we need to compare the financing and investment allocation costs of both strategies.

If the FI induces the UI to continue, the FI must increase $X$ to $\frac{\delta I+(1-\delta) L}{\sigma_{b}}$, which guarantees the UI the same interim expected payoff as that from withdrawal. Note that even in this case, the initial security is not necessarily safe; thus, $X$ must be adjusted for the risk.

If the FI induces the UI to withdraw, the FIm must finance $I$ by issuing the new security with $Y^{*}=\frac{\sigma_{\ell} R}{\sigma_{\ell}+\left(1-\sigma_{\ell}\right) \sigma_{Z}}$ at time 1 to separate itself from the FI $\ell$. Note that $Y^{*}$ is adjusted for outside investors' premiums. On the other hand, the FI $\ell$ liquidates $A_{I}$ and pays the UI the liquidation value $L$. As $L$ is greater than the expected cash flow in continuation, $\sigma_{\ell} R$, under Assumption 1, the FI can reduce the face value of the initial security $X$ by inducing the UI 
to withdraw and by repaying the UI the certain value of $L$ instead of $\sigma_{\ell} X$ when $q=\ell$.

Comparing the costs of the above two strategies, we show that if $\frac{R}{I}$ becomes larger or $L$ becomes smaller, the face value of the initial security inducing the UI to continue is decreasing relative to the face value of the initial security inducing the UI to withdraw, whereas the new security payment of the FI inducing the UI to withdraw is increasing relative to $I$. Thus, the cost of inducing the UI to continue is more likely to be lower than the cost of inducing the UI to withdraw, as $\frac{R}{I}$ is increasing relative to $L$. Given the definition of $\psi(\mathcal{L})$, this implies that in the range of $\frac{R}{I} \in\left[\Re_{2}, \Re_{1}\right)$, the FI induces the UI to continue at $s=b$ if the profitability of $A_{I}$ at success is moderate but is still high enough relative to $\mathcal{L}\left(\Re_{1}>\frac{R}{I} \geq \psi(\mathcal{L})\right)$; in contrast, the FI induces the UI to withdraw at $s=b$ if the profitability of $A_{I}$ at success is moderate but is not high enough relative to $\mathcal{L}\left(\psi(\mathcal{L})>\frac{R}{I} \geq \Re_{2}\right)$.

Finally, suppose that the profitability of $A_{I}$ at success is sufficiently low $\left(\Re_{2}>\frac{R}{I}>\frac{1}{\sigma_{m}}\right)$. Then, the argument is similar to that in the case of $\psi(\mathcal{L})>\frac{R}{I} \geq \Re_{2}$. In addition, as discussed in Lemma 1(iii), the early withdrawal of the UI causes no adverse selection problem in the ex post capital market in this range of $\frac{R}{I}$. Thus, the FI $m$ need not repay any premiums to finance the withdrawn amount.

\section{The Optimality of Nonrecourse Financing}

In Section 3, we assumed that the FI can observe the quality of $A_{I}$ at the end of time 0 . Then, Lemma 4 indicates that there may exist a region in which the UI is induced not to withdraw at $s=b$. In this section, we first check whether this region can survive if the FI can choose whether to undertake information acquisition activity. The optimality of nonrecourse financing can be derived using this result.

\subsection{Informed vs. uninformed finance.-}

We suppose that the FI cannot observe the quality of $A_{I}$ at the end of time 0 unless it pays a positive cost $\epsilon_{I}$ to acquire information at the beginning of time 0 . This is because the FI must develop methodologies and skills and collect information about the borrower to evaluate the quality of $A_{I}$. For simplicity, we assume that $\epsilon_{I}$ is an infinitesimally small positive number, and that the UI and outside investors know whether information acquisition activity is undertaken. ${ }^{23}$ Although $\epsilon_{I}$ is not small, the main results in this paper are unaffected.

\footnotetext{
${ }^{23}$ This assumption is also used in Winton (2003). As an alternative, and as in Repullo and Suarez (1998), we may assume that the FI can (contractually) commit to using information technology.
} 
Because the informed finance equilibrium (IFE) where the FI engages in information acquisition is exactly the same as that explored in Section 3, except that the FI pays $\epsilon_{I}$ at time 0 , we need only investigate the uninformed finance equilibrium (UFE) where the FI engages in no information acquisition. We then obtain the following lemma.

Lemma 5: Suppose that the FI does not undertake any information acquisition activity.

(i) Suppose that $\frac{1}{\sigma_{\ell}}>\frac{R}{I} \geq \frac{1}{\sigma_{b}}$. The optimal strategies of the FI and the UI under the UFE are the same as those under the IFEP given by Lemma 4 (i).

(ii) Suppose that $\frac{1}{\sigma_{b}}>\frac{R}{I}>\frac{1}{\sigma_{m}}$. Let $\Pi_{F 0}$ denote the expected payoff the FI at the beginning of time 0 . Then, the optimal value of $\Pi_{F 0}$ is lower than or equal to $\widehat{\Pi}_{F 0}$, where $\widehat{\Pi}_{F 0} \equiv[p+$ $\left.(1-p) \sigma_{b}\right] R+\omega Z-\bar{I}$.

If the profitability of $A_{I}$ at success is not substantially low (as in the case of Lemma $5(\mathrm{i})$ ), the UFE definitely causes overinvestment at $s=b$, in the sense that the FI raises new funds and continues even when $q=\ell$. Further, in the range $\frac{R}{I}$, the UFE is the same as the IFEP given in Lemma 4(i), except that the FI does not pay $\epsilon_{I}$ for information acquisition. This implies that despite the nonrecourse security, the uninformed FI may provide inefficient liquidity support at $s=b$ after the UI withdraws. On the other hand, when the profitability of $A_{I}$ at success is substantially low (as in the case of Lemma 5(ii)), the optimal value of the expected payoff the FI at the beginning of time $0, \Pi_{F 0}$, is lower than or equal to $\widehat{\Pi}_{F 0}$, which would be attained if the FI were able to commit to continue after the withdrawal of the UI.

The intuition for Lemma 5 is as follows. In the case of Lemma 5(i), the uninformed FI prefers to continue at $s=b$, regardless of whether the UI continues or withdraws. The optimal strategies of the FI and the UI in this case are then the same as those under the IFEP of Lemma 4(i) because in this range of $\frac{R}{I}$, the FI does not use any information on $q$, even though it acquires information. In the case of Lemma 5(ii), the withdrawal of the UI at $s=b$ always forces the uninformed FI to liquidate $A_{I}$ and receive nothing. Hence, the optimal value of $\Pi_{F 0}$ is lower than or equal to $\widehat{\Pi}_{F 0}$.

Inspecting the ex ante expected payoff of the FI attained in each case of Lemmas 4 and 5, we establish the following lemma about the configuration of the IFE and the UFE.

Lemma 6: Define $\phi(\mathcal{L}) \equiv[\delta+(1-\delta) \mathcal{L}] \Re_{1}$.

(i) If $\frac{1}{\sigma_{\ell}}>\frac{R}{I} \geq \max \left(\phi(\mathcal{L}), \Re_{2}\right)$, the equilibrium is given by the UFE that is characterized by Lemma $5(i)$.

(ii) If $\max \left(\phi(\mathcal{L}), \Re_{2}\right)>\frac{R}{I} \geq \frac{1}{\sigma_{m}}$, the equilibrium is given by the IFES, which is characterized 


\section{by Lemma 4(iii).}

Figure 2 illustrates the configuration of the equilibrium given by Lemma 6 with various regions of the parameter space (as depicted by the parameters $\mathcal{L}$ and $\frac{R}{I}$ ) where the UFE or the IFES prevails. ${ }^{24}$

The intuition for this lemma is as follows. Under Assumption 5(ii), note that $\Re_{2}>\frac{1}{\sigma_{b}}$.

Suppose that the profitability of $A_{I}$ at success is sufficiently high $\left(\frac{1}{\sigma_{\ell}}>\frac{R}{I} \geq \Re_{1}\right)$. In this case, regardless of whether or not the FI acquires information, it always sets $X^{*}=I$ and continues to invest in $A_{I}$ although the UI is induced to withdraw the fund at $s=b$ (see Lemmas $4(\mathrm{i})$ and $5(\mathrm{i})$ ). The reason is that investment in $A_{I}$ is worth continuing because $\frac{R}{I}$ is sufficiently high and the FI receives nothing in liquidation. As the information obtained by the FI need not be used in the range of $\frac{R}{I}$, the FI does not acquire information in order to save the information acquisition cost. As a result, uninformed finance prevails.

Next, suppose that the profitability of $A_{I}$ at success is moderate but relatively higher than $\mathcal{L}\left(\Re_{1}>\frac{R}{I} \geq \psi(\mathcal{L})\right)$. If the FI acquires information in this range, it is always optimal for the FI to induce the UI to continue investment because $\frac{R}{I}$ is relatively higher than $L$ and the FI receives nothing in liquidation (see Lemma 4(ii)). As a result, the FI must raise the face value of the initial security, $X$, to compensate the UI for the default risk borne when the UI continues to invest. Under uninformed finance, however, the FI can commit to induce the UI to withdraw the fund so that the FI need not pay the increment in $X$ (see Lemma 5(i)). Under Assumption 5(i), we can show that the possibility of the FI inducing the UI to continue under informed finance is ruled out by uninformed finance.

We now suppose that the profitability of $A_{I}$ at success is moderate but is not as high as $\mathcal{L}\left(\psi(\mathcal{L})>\frac{R}{I} \geq \Re_{2}\right)$. In this case, whether or not informed finance prevails depends on the benefit and cost of the separating strategy chosen by the FI after it observes $q$ at $s=b$ (see Lemma 4(iii)). The separating strategy creates the benefit of the efficient investment for both $q=m$ and $q=\ell$ because the FI always induces the UI to withdraw the fund at $s=$ $b$, and raises new funds and provides liquidity support only if $q=m$. On the other hand, there are two costs in the separating strategy. The FI needs to determine the face value of the initial security, $X$, by considering that the FI $m$ continues to invest in $A_{I}$ while the FI $\ell$ liquidates $A_{I}$. Given $L<I$, this implies that the separating strategy sets $X$ higher than

\footnotetext{
${ }^{24}$ Given that $\phi(1)=\Re_{1}, \phi^{-1}\left(\Re_{2}\right)>\frac{\sigma_{\ell}}{\sigma_{m}}$ and $\phi\left(\mathcal{L}^{\circ}\right)=\Re_{2}$, where $\frac{\mathcal{L}^{\circ}}{\sigma_{\ell}}=\phi\left(\mathcal{L}^{\circ}\right)$, Figure 2 is drawn by assuming $\mathcal{L}^{\circ}<\frac{\sigma_{b}}{\sigma_{m}}$. In addition, note that $\frac{R}{I}=\phi(\mathcal{L})$ is included in the region $\left\{\left(\mathcal{L}, \frac{R}{I}\right) \mid \frac{1}{\sigma_{b}} \mathcal{L}<\frac{R}{I}<\frac{1}{\sigma_{\ell}} \mathcal{L}\right.$ and $\Re_{2}<\frac{R}{I}$ $\left.<\Re_{1}\right\}$ for $\mathcal{L} \in\left(\mathcal{L}^{\circ}, 1\right)$. This is because $\phi^{\prime}(\mathcal{L})=(1-\delta) \Re_{1} \in\left(0, \frac{1}{\sigma_{\ell}}\right), \phi\left(\mathcal{L}^{\circ}\right)=\Re_{2}$ and $\phi(1)=\Re_{1}$.
} 
$I$ in this region. Furthermore, the FIm must repay outside investors' premiums in excess of the fair risk-adjusted level to separate itself from the FI $\ell$ in the ex post capital market. The benefit of the efficient investment, however, is more likely to outweigh the costs of the increments of the initial face value and the repayment in the ex post capital market when $\mathcal{L}$ is large or $\frac{R}{I}$ is small. ${ }^{25}$ Hence, the FI undertakes information acquisition when $\mathcal{L}$ is large or $\frac{R}{I}$ is small.

Finally, if the profitability of $A_{I}$ at success is sufficiently low $\left(\Re_{2}>\frac{R}{I} \geq \frac{1}{\sigma_{m}}\right)$, the FIm need not pay any premiums to finance the withdrawn funds in the ex post capital market (see Lemma 4(iii)). Informed finance always prevails in this region because the benefit of the efficient investment always outweighs the incremental cost of the initial face value.

Using Lemmas 4-6 with Figure 2, we now establish the following proposition.

Proposition 1: If the FI can choose whether to undertake information acquisition activity, the equilibrium is characterized as follows.

(i) The FI always offers an initial security that induces the UI to withdraw when $s=b$.

(ii) The FI undertakes no information acquisition, provides inefficient liquidity support, and overinvests if the profitability of $A_{I}$ at success is not sufficiently small relative to $\mathcal{L}\left(\frac{1}{\sigma_{\ell}}>\frac{R}{I}\right.$ $\left.\geq \max \left(\phi(\mathcal{L}), \Re_{2}\right)\right)$. Otherwise, the FI undertakes information acquisition, provides efficient liquidity support, and invests efficiently.

Proposition 1 suggests that if the FI can choose whether to acquire information, the optimal initial security always induces the UI to withdraw early at $s=b$ and forces the FI to determine whether to raise new funds in order to give liquidity support. The intuition is that if the FI commits to acquire information, there is a region of $\left(\mathcal{L}, \frac{R}{I}\right)$ where the UI does not prefer to withdraw early at $s=b$ because the FI $\ell$ then liquidates $A_{I}$. In this region, the FI needs to increase the face value of the initial security to compensate the UI for the default risk arising when the UI continues. However, in this region, if the FI does not attempt to acquire information, the FI can commit to pay the UI the certain redemption amount of $I$, raise new funds, and continue investment in $A_{I}$ after the withdrawal of the UI. Then, the FI can avoid compensating the UI for the default risk arising when the UI continues. Thus, if the FI can choose whether to acquire information, in this region, the FI prefers not to acquire information, and induces the UI to withdraw early at $s=b$ to prevent the face value

\footnotetext{
${ }^{25} \frac{R}{I}=\phi(\mathcal{L})$ is a threshold line below which the former benefit outweighs the latter costs. Note that $\varphi(\mathcal{L})$ $>\phi(\mathcal{L}), \phi(1)=\Re_{1}$, and $\phi^{\prime}(\mathcal{L})>0$. Thus, $\min \left(\varphi(\mathcal{L}), \Re_{1}\right)>\phi(\mathcal{L})$ for any $\mathcal{L} \in(0,1)$.
} 
of the initial security from increasing.

Proposition 1 also indicates that if the profitability of $A_{I}$ at success is not sufficiently small relative to $\mathcal{L}\left(\frac{1}{\sigma_{\ell}}>\frac{R}{I} \geq \max \left(\phi(\mathcal{L}), \Re_{2}\right)\right)$, inefficient maturity mismatch (inefficient early withdrawal) arises. Despite the nonrecourse security, the FI $\ell$ provides liquidity support and continues to invest in $A_{I}$. Intuitively, as the FI does not undertake information acquisition activity in the region, it does not know its own quality. However, the uninformed FI can raise sufficient funds for the devalued $A_{I}$ at $s=b$. Thus, there is inefficient liquidity support and overinvestment for $q=\ell$ when $s=b$. Conversely, if the profitability of $A_{I}$ at success is not sufficiently small relative to $\mathcal{L}\left(\max \left(\phi(\mathcal{L}), \Re_{2}\right)>\frac{R}{I}>\frac{1}{\sigma_{m}}\right)$, the optimal initial security provides a liquidity threat that not only leads to efficient liquidity support and investment decision by the FI but also enhances information acquisition by the FI.

According to Calomiris and Kahn (1991), Flannery (1994), and Diamond and Rajan (2001), the mismatch in the maturity structure or the possibility of the early withdrawal of lenders serves as a commitment device to discipline commercial banks. These studies show that demand deposits are a way of reassuring investors in a commercial bank that their investments will not be too badly expropriated by bank management. Conversely, Stein (2005) finds that mutual and hedge funds allow early withdrawals (equivalent to open ending) in an attempt to signal the skill of the funds' managers. However, because both high-quality and low-quality managers are forced to open end, the resulting equilibrium is pooling, where all funds choose an open-end form and an inefficient investment strategy. Brunnermeier and Oehmke (2010) also derive the optimality of excessively short-term financing under the possibility of early withdrawal of lenders, although short-term financing forces lenders to be exposed to unnecessary liquidation.

In contrast to the existing literature, in our model, whether the maturity mismatch causes inefficiency depends on the parameter values of $\frac{R}{I}$ and $\mathcal{L}$. If the profitability of $A_{I}$ at success is not sufficiently small relative to $\mathcal{L}\left(\frac{1}{\sigma_{\ell}}>\frac{R}{I} \geq \max \left(\phi(\mathcal{L}), \Re_{2}\right)\right)$, the maturity mismatch causes inefficient liquidity support and overinvestment because the equilibrium is pooling, as indicated in Stein (2005). Otherwise, the maturity mismatch brings about efficient liquidity support and investment because it serves as a commitment device to discipline the FI, as suggested in Calomiris and Kahn (1991), Flannery (1994), and Diamond and Rajan (2001). Although the Brunnermeier and Oehmke (2010) model causes excessive liquidation, this result depends on their rat-race framework in which, given that all other lenders are only providing short-term financing, it is not individually rational for the financial institution to 
move an individual creditor to a longer maturity. Further, in their setting, there exists no ex post capital market after the withdrawal of creditors.

\subsection{Optimality of the initial security backed by the cash flow of $A_{I}$ alone.-}

We initially assumed that the FI could pledge only the cash flow of $A_{I}$ at time 0 . To justify this, we assumed that prior to time 0, the FI issued the old security backed by the cash flow of $A_{O}$ to finance the FI's other urgent or opaque liquidity needs. In its place, the FI may repurchase the existing old security issued prior to time 0 and sell at time 0 an initial security backed by the cash flows of $A_{I}$ and $A_{O}$ to raise both $I$ and the repurchase payment. This is equivalent to assuming that the FI can pledge the cash flows of both $A_{I}$ and $A_{O}$ at time 0 . This type of initial security issued at time 0 and backed by the cash flows of $A_{I}$ and $A_{O}$ can be regarded as an alternative to the initial security issued at time 0 and backed by the cash flow of $A_{I}$ alone, which has been considered in the previous discussions.

If the FI issues this initial security at time 0 backed by the cash flows of $A_{I}$ and $A_{O}$ to raise both $I$ and the repurchase payment, we can assume that the initial security matures at time 1 and that the FI decides whether to roll it over at time 1. As the FI's other urgent or opaque liquidity needs disappear at time 1 , the FI only needs to finance the amount $I$ when rolling over the initial security at time 1. The initial security introduced above is then essentially equivalent to the security offered by the FI to the UI at the beginning of time 0 so that the UI provides $I$ and requires a repayment $X$ at time 2 if $A_{I}$ and $A_{O}$ yield a cash flow $R+Z$, a repayment $X^{\prime}$ at time 2 if $A_{I}$ and $A_{O}$ yield $Z$, and no repayment at time 2 if $A_{I}$ and $A_{O}$ yield no cash flow. Note that $R+Z \geq X \geq 0$ and $Z \geq X^{\prime} \geq 0$.

Under this type of initial security, suppose that the UI withdraws and the FI liquidates $A_{I}$ at time 1 . However, the liquidation value of $A_{I}, L$, cannot cover the redemption amount, $I$, under Assumption 1. Thus, when the FI pledges the cash flows of both $A_{I}$ and $A_{O}$, it must liquidate $A_{O}$ to repay the remaining amount $I-L$ unless it raises new funds in the ex post capital market. In fact, the liquidation value of $A_{O}$ equals 0 , whereas the expected cash flow of $A_{O}$ at $s=b$ is larger than $I-L$ even for $q=\ell$ if $\left[\sigma_{\ell}+\left(1-\sigma_{\ell}\right) \sigma_{Z}\right] Z>I-L$. This last condition is satisfied under Assumption 4. Thus, when the FI liquidates $A_{I}$ at time 1, it prefers to avoid the liquidation of $A_{O}$ because it cannot receive anything if it liquidates $A_{O}$. Hence, the FI must issue at time 1 a new security backed by the cash flow of $A_{O}$ and finance $I-L$ to avoid the liquidation of $A_{O}$.

Given these modifications, repeating the arguments for $s=g$ at the beginning of Section 3.1 
and the arguments in Sections 3.1.1 and 3.1.2, we show the following lemma that corresponds to Lemmas 1 and 2.

Lemma 7: Suppose that the FI undertakes information acquisition activity. Under the initial security backed by the cash flows of both $A_{I}$ and $A_{O}$, the optimal strategies of the FI at time 1 are characterized as follows.

(i) If the UI continues to invest in $A_{I}$ at time 1 , the FI continues to invest in $A_{I}$ for any $s$.

(ii) Suppose that the UI withdraws at time 1.

(a) Suppose that $s=g$. Then, the FI continues to invest in $A_{I}$.

(b) Suppose that $s=b$. ( $\alpha$ ) If $\frac{1}{\sigma_{\ell}}>\frac{R}{I} \geq \Re_{1} \mathcal{L}$, both the FIm and the FI $\ell$ continue to invest in $A_{I}$. The repayment level from the FI to outside investors in the capital market is $Y^{P}$ $=\frac{I}{\sigma_{b}+\left(1-\sigma_{b}\right) \sigma_{Z}}$. ( $\left.\beta\right)$ If $\Re_{1} \mathcal{L}>\frac{R}{I} \geq \frac{1}{\sigma_{m}}$, the FIm continues to invest in $A_{I}$ while the FI liquidates $A_{I}$. The repayment level from the FIl to outside investors in the capital market is $Y^{\ell}=\frac{I-L}{\sigma_{\ell}+\left(1-\sigma_{\ell}\right) \sigma_{Z}}$. The repayment level from the FIm to outside investors in the capital market is $Y_{R+Z}^{m}=Y_{Z}^{m}=Y^{m}: Y^{m}=\frac{\sigma_{\ell} R+I-L}{\sigma_{\ell}+\left(1-\sigma_{\ell}\right) \sigma_{Z}}$ if $\Re_{1} \mathcal{L}>\frac{R}{I} \geq \max \left(\Re_{2}-\frac{1-\mathcal{L}}{\sigma_{\ell}}, \frac{1}{\sigma_{m}}\right)$; and $Y^{m}=\frac{I}{\sigma_{m}+\left(1-\sigma_{m}\right) \sigma_{Z}}$ if $\max \left(\Re_{2}-\frac{1-\mathcal{L}}{\sigma_{\ell}}, \frac{1}{\sigma_{m}}\right)>\frac{R}{I}>\frac{1}{\sigma_{m}}$.

Because $1>\mathcal{L}$, the possibility of both the FIm and the FI $\ell$ raising new funds and continuing to invest in $A_{I}$ after the withdrawal of the UI at $s=b$ is more likely to occur if the initial security is backed by the cash flows of both $A_{I}$ and $A_{O}$ instead of $A_{I}$ alone. Intuitively, even if the FI liquidates $A_{I}$ at time 1 when the initial security is backed by both $A_{I}$ and $A_{O}$, it does not prefer to liquidate $A_{O}$ to repay the insufficient amount $I-L$. Thus, the FI issues the new security backed by $A_{O}$ and finances $I-L$. As the probability of $A_{O}$ yielding $R$ is larger for $q=m$ than for $q=\ell$, this new security issue further strengthens the FI $\ell$ 's imitating incentive, thus making it more expensive for the FI $m$ to separate itself from the FI . As a result, under the recourse security, the pooling equilibrium strategy arises in a broader region after the withdrawal of the UI because it is more costly for the FI $\ell$ to liquidate $A_{I}$. Backing the security with other assets of the FI is thus more likely to induce the FI $\ell$ to continue investing in $A_{I}$ at time 1 after the UI withdraws. As shown below, this implies that the recourse security would raise the FI's borrowing costs when the FI can choose whether to acquire information.

Next, applying the arguments for $s=g$ at the beginning of Section 3.1 and the arguments in Section 3.1.3, and using the results of Lemma 7, we show the following lemma that corresponds to Lemma 3. 
Lemma 8: Suppose that the FI undertakes information acquisition activity. Under the initial security backed by the cash flows of both $A_{I}$ and $A_{O}$, the optimal strategies of the UI at time 1 are presented as follows.

(i) Suppose that $s=g$. Then, the UI always continues.

(ii) Suppose that $s=b$. Then, the UI continues if $\sigma_{b} X+\left(1-\sigma_{b}\right) X^{\prime} \geq I$. Otherwise, the UI withdraws.

Note that the UI receives $I$ even at the liquidation of $A_{I}$ because the FI must avoid the liquidation of $A_{O}$ in this security. Hence, the UI can always obtain $I$ when withdrawing.

Now, using arguments similar to those of Lemma 4, the configuration of the IFE under the initial security backed by both $A_{I}$ and $A_{O}$ is given by a form similar to the configuration of the IFEP, IFEC, and IFES indicated by Lemma 4. In fact, for the region corresponding to the IFEC of Lemma 4, recourse financing may be optimal. Intuitively, if the initial security is backed by the cash flows of both $A_{I}$ and $A_{O}$ instead of $A_{I}$ alone, it reduces the bankruptcy possibility at time 2 if the UI is induced to continue investing when $s=b$ (as in the region corresponding to the IFEC of Lemma 4). Such a reduction of the bankruptcy possibility at time 2 relaxes the incentive compatibility constraint to ensure that the UI continues, thereby leading to a reduction of the FI's initial promised repayment when $s=g$ and lowering its borrowing costs. Thus, if the FI need not raise additional funds in the capital market at $s=b$ because the UI continues investing at $s=b$, the decline in the FI's borrowing costs may make recourse financing advantageous.

To exclude the above possibility, we again assume that the FI can choose whether to undertake information acquisition activity. First, repeating arguments similar to those used to derive Lemma 5, we have the following lemma:

Lemma 9: The optimal value of $\Pi_{F 0}$ under uninformed finance when an initial security is backed by the cash flows of both $A_{I}$ and $A_{O}$ is equal to $\widehat{\Pi}_{F 0}$ if $\frac{R}{I} \geq \frac{\mathcal{L}}{\sigma_{b}}$; otherwise, it is lower than or equal to $\widehat{\Pi}_{F 0}$, where $\widehat{\Pi}_{F 0} \equiv\left[p+(1-p) \sigma_{b}\right] R+\omega Z-\bar{I}$.

Intuitively, under uninformed finance in this case, the FI continues after the withdrawal of the UI if and only if $\sigma_{b} R-I \geq-(I-L)$; that is, $\sigma_{b} R \geq L$. This is because the FI must raise the amount $I-L$ to avoid the liquidation of $A_{O}$ when liquidating $A_{I}$. Then, repeating arguments similar to those used in Lemma 5, we obtain the result of this lemma.

Using Lemmas 7-9, we obtain the following proposition.

Proposition 2: If the FI can choose whether to undertake information acquisition activity, 
the initial security backed by the cash flow of $A_{I}$ alone dominates the initial security backed by the cash flows of both $A_{I}$ and $A_{O}$.

Intuitively, the initial security backed by both $A_{I}$ and $A_{O}$ makes it more difficult for the FI $\ell$ to liquidate $A_{I}$ at $s=b$. The reason is that if the FI liquidates $A_{I}$, it must issue the new security backed by $A_{O}$ and finance $I-L$ to avoid the liquidation of $A_{O}$. However, this strengthens the FI $\ell$ 's imitating incentive, which forces the FI $m$ to issue the more underpriced security to separate itself from the FI $\ell$. On the other hand, because the initial security backed by $A_{I}$ alone makes $A_{I}$ remote from $A_{O}$, it enables the FI to avoid the liquidation of $A_{O}$ without the issue of any new security when liquidating $A_{I}$. This makes liquidation of $A_{I}$ more attractive for the FI $\ell$, which knows that $A_{I}$ is bad. In fact, the initial security backed by both $A_{I}$ and $A_{O}$ can reduce the bankruptcy possibility at time 2 relative to the initial security backed by $A_{I}$ alone if the UI continues investment at $s=b$. This effect reduces the expected initial promised repayment of the FI by relaxing the incentive compatibility constraint that ensures that the UI continues. However, the latter effect does not exist as long as the UI withdraws at $s=b$. The reason is that the individual rationality constraint to ensure that the UI can recover its funding amount $I$ must then be binding, instead of the incentive compatibility constraint to ensure that the UI withdraws. Then, the expected initial promised repayment of the FI cannot be reduced because it must be set equal to $I$.

Hence, if we compare the benefits of the recourse security with those of the nonrecourse security, the benefits of reducing the bankruptcy possibility under the recourse security are dominated by those of relaxing the FI $\ell$ 's imitating incentives under the nonrecourse security. Thus, the commitment provided by recourse financing is harmful because it affords the FI $\ell$ more incentives to continue investing in $A_{I}$ at $s=b$ and causes the inefficient allocation of investment, although it may reduce the possibility of bankruptcy at time 2 . Thus, the FI's ex ante expected payoff will be lower under the recourse security. By establishing bankruptcy remoteness, the nonrecourse security allows the FI to commit to more efficient decisions on whether to liquidate or continue $A_{I}$. As a result, even though the FI may finance additional funds and provide liquidity support to $A_{I}$ afterward, the nonrecourse security becomes optimal in the first place.

This logic is similar to that of Inderst and Mueller (2010), who examine the optimal CEO compensation and replacement policy when the CEO privately observes an interim signal about the likely firm value under his continued leadership and when the CEO turnover must 
be incentive compatible from the CEO's perspective. The CEO's desire to continue to hold his position, together with his private information at the interim stage, creates a wedge between efficient CEO replacement and actual CEO turnover. Steep incentive and severance pay can mitigate such CEO entrenchment because it makes continuation unattractive for "bad" CEOs. The nonrecourse security in our model plays a role similar to that of the steep incentive and severance pay in Inderst and Mueller, in the sense that it makes continuation of investment in $A_{I}$ unattractive for the FI $\ell$.

Proposition 2 reveals a novel aspect of the security design of the financial institution: namely, the institution should initially issue a security backed by a particular investment asset instead of its total assets to resolve the overinvestment problem. This novel aspect is crucially dependent on three features: (i) the possibility of maturity mismatch (or early withdrawal), (ii) information asymmetry, and (iii) the endogenous choice of information acquisition. Note that the FI prefers recourse financing unless the first two features exist. The reason is that recourse financing then does not strengthen the FI $\ell$ 's imitating incentive, but reduces the FI's borrowing costs through a reduction in the bankruptcy possibility at time 2. Further, if the FI commits to acquiring information, nonrecourse financing may not be optimal for $\frac{R}{I} \in\left[\psi(\mathcal{L}), \Re_{1}\right)$, when the UI is induced to continue investment in $A_{I}$ at $s=$ $b$ (see Lemma 4(ii)). This is because, in this case, the FI need not raise additional funds at $s=b$. Again, recourse financing does not tighten the FI $\ell$ 's imitating incentive, but reduces the FI's borrowing costs through a decrease in the bankruptcy possibility at time 2 .

Benveniste and Berger (1987) and Carlstrom and Samolyk (1995) indicate that either the exogenous recourse or the capital constraint on the original bank is a key factor in explaining the role of asset-backed lending. On the other hand, the logic of Proposition 2 makes it clear that the optimality of nonrecourse financing of securitization is endogenously derived and is interrelated with not only the maturity structure of the FI and the information asymmetry, but also the FI's information acquisition activity.

Ayotte and Gaon (2011) discuss securitization by focusing on the special protection provided by 'bankruptcy remoteness' to distinguish asset-backed securities (ABS) from secured or unsecured debt. They suggest that in bankruptcy, the debtor-in-possession (DIP) lender can transfer sufficient wealth from existing unsecured creditors through dilution of their claims by issuing senior claims. Ayotte and Gaon show that ABS protect ABS investors from the DIP lender in bankruptcy and can prevent inefficient continuation of the firm. However, they do not allow ABS investors to withdraw early, nor do they examine the ad- 
verse selection issues. Proposition 2 highlights the role of the nonrecourse security that mitigates the adverse selection incentive for the FI $\ell$ in the ex post capital market when the UI withdraws early. ${ }^{26}$

DeMarzo and Duffie (1999) and DeMarzo (2005) argue that the process of pooling and tranching in securitization alleviates the underpricing involved in the asset sale, because it enhances risk diversification and prevents informed intermediaries from exploiting uninformed investors under the ex post signaling model. In fact, these papers do not consider the early withdrawal of uninformed investors, nor do they consider the additional financing possibility of informed intermediaries. Alternatively, Proposition 2 suggests that the nonrecourse property of securitization mitigates the underpricing involved in the additional financing by the FI after the UI withdraws.

Gorton and Souleles (2006) discuss the possibility of liquidity support by a sponsor for its SPV in securitization in certain states of the world, even though it is not legally bound to do so. They suggest that the sponsor colludes with investors in the SPV by agreeing to the implicit state-contingent subsidization in a repeated game context. In this paper, the FI provides liquidity support for $A_{I}$, even in a static context if it is ex post optimal for the FI to do so when the UI withdraws at time 1. However, if the profitability of $A_{I}$ at success is not sufficiently small relative to $\mathcal{L}\left(\frac{1}{\sigma_{\ell}}>\frac{R}{I} \geq \max \left(\phi(\mathcal{L}), \Re_{2}\right)\right)$, inefficient liquidity support and overinvestment occur, even in the case of the nonrecourse security.

Combining Propositions 1 and 2, we establish the following corollary.

Corollary to Propositions 1 and 2: If the FI can choose whether to undertake information acquisition activity, the nonrecourse financing of securitization dominates traditional bank loans with deposit claims backed by the bank's total assets. However, inefficient liquidity support and overinvestment arise despite the nonrecourse property of the security, as long as the profitability of $A_{I}$ at success is not sufficiently small relative to $\mathcal{L}$.

To summarize, the nonrecourse financing of securitization may be used even though not only regulatory and ratings arbitrage, but also the low-interest-rate environment are alle-

\footnotetext{
${ }^{26}$ Proposition 2 also provides a different aspect of securitization from Bolton and Freixas (2000). They assume the priority of bank debt over bonds and suggest that the priority structure invokes excessive liquidation because the bank's priority can be maintained only if the firm is liquidated. Securitization in their model arises to remove the undesirable effects of priority structure by making the bank's claim junior to the claims of asset-backed security holders. By contrast, in our model, the priority structure creates the overinvestment problem, as in Ayotte and Gaon (2011). Proposition 2 suggests that under adverse selection problems, asset-backed securities with a nonrecourse property are more likely to motivate the institution to liquidate its deteriorated assets, and are thus more likely to alleviate the overinvestment problem.
} 
viated. This result also holds notwithstanding that the FI may provide liquidity support afterward.

\subsection{Comparative statics.-}

We conduct a comparative static analysis. The variables in which we are interested are the profitability of $A_{I}$ at success, $\frac{R}{I}$, the ratio of the liquidation value of $A_{I}$ to the funding amount of the UI, $\mathcal{L}$, the asset quality of $A_{I},\left(\sigma_{m}, \sigma_{\ell}\right)$, and the asset quality of $A_{O}, \sigma_{Z}$.

Proposition 3: If the FI can choose whether to undertake information acquisition activity, the UFE - where the FI undertakes no information acquisition, provides inefficient liquidity support, and overinvests - is more likely to occur, the larger is $\frac{R}{I}$, the smaller is $\mathcal{L}$, the larger is $\sigma_{m}$, the larger is $\sigma_{\ell}$, or the smaller is $\sigma_{Z}$.

Proposition 3 suggests that the UFE is more likely to occur when the profitability of $A_{I}$ at success increases or when the liquidation value of $A_{I}$ decreases relative to the funds raised from the UI or when the asset quality of $A_{I}$ (or $A_{O}$ ) is expected to be higher (lower).

The intuition for this proposition is as follows. The results of $\frac{R}{I}$ and $\mathcal{L}$ depend on the FI $\ell$ continuing (or liquidating) at $s=b$ in the UFE (or the IFES) in Lemma 6. This implies that an increase in $R$ increases the ex ante expected payoff of the FI, $\Pi_{F 0}^{*}$, in the UFE more than $\Pi_{F 0}^{*}$ in the IFES. Hence, the FI is less likely to acquire information, the larger is $\frac{R}{I}$. By contrast, when $L$ is smaller, $\Pi_{F 0}^{*}$ in the IFES decreases while $\Pi_{F 0}^{*}$ in the UFE is unchanged. Hence, the FI is less likely to acquire information, as $\mathcal{L}$ is smaller. A rise in $\sigma_{m}$ or $\sigma_{\ell}$ increases $\Pi_{F 0}^{*}$ in the UFE more than $\Pi_{F 0}^{*}$ in the IFES. This is because a rise in $\sigma_{m}$ or $\sigma_{\ell}$ reduces the FI's repayment to outside ex post capital market investors in the UFE by making it easier for the FI to assure outside investors of their reservation payoff, whereas a rise in $\sigma_{\ell}$ increases the FIm's repayment in the IFES by making it more difficult for the FI $m$ to separate itself from the FI $\ell$. On the other hand, an increase in $\sigma_{Z}$ decreases the FI's repayment to outside investors in the IFES more than that in the UFE by mitigating the adverse selection problem. This reduces the likelihood of the UFE.

\section{Empirical Implications}

\subsection{Securitized and structured finance.-}

Asset-backed securities are one of the most significant financial innovations of recent decades. In particular, a growing number of commercial and investment banks issue struc- 
tured finance products such as MBSs and CDOs into which mortgages and other loans are pooled. The portfolios of the products are usually transferred to off-balance-sheet vehicles. In these products, the securitized claims on the pool's payments are carved into various 'tranches' or classes of risk, for example, senior, mezzanine and equity tranches. The majority of buyers of senior tranches are institutional investors, while parts of the lower tranches are sold to high-yield-seeking investors, such as hedge funds. Recently, in order to sell some parts of the senior tranches, banks have acted as sponsors of ABCP programs by letting off-balance-sheet vehicles-ABCP conduits and SIVs - issue short-term maturity paper in the form of $\mathrm{ABCP} .^{27}$

As ABCP conduits invest in illiquid long-maturity assets and issue short-maturity paper, sponsoring banks typically provide credit enhancement and/or liquidity support to their conduits. Indeed, the majority of $\mathrm{ABCP}$ conduits have liquidity lines committed by sponsoring banks for the full amount of outstanding CP. Hence, if the ABCP conduits face liquidity needs such that their issued CP is to be forcibly repurchased, they will need to be funded by the sponsoring banks. SIVs are similar to ABCP conduits. The difference is that SIVs operate with much less liquidity support and have substantially more stringent rules of operation. ${ }^{28}$ In fact, sponsoring banks are often forced to support the SIV financially even though they are legally separate entities. As a result, ABCP investors are in most cases insulated from market value risk. If an ABCP conduit or SIV cannot roll over its maturing paper, and liquidity drawdowns are thus made to the full extent of the CP outstanding, the sponsoring bank is forced to provide funding support and becomes the sole funding provider. Because a large number of agents are involved in ABCP programs and are unknown to the sponsoring banks, the renegotiation of ABCP programs is practically impossible. ${ }^{29}$

In our model, the sponsoring bank, ABCP investors, and MBSs and CDOs or their original assets are interpreted as the FI, the UI, and the investment asset $A_{I}$, respectively. Offbalance-sheet vehicles and their financing strategy of borrowing using short-term paper are also viewed as the nonrecourse and short-maturity properties of the initial security issued by

\footnotetext{
${ }^{27} \mathrm{ABCP}$ conduits are bankruptcy-remote SPVs established by sponsoring banks and are funded through the issuance of ABCP to finance the purchase of MBSs and CDOs. An SPV is a legal entity created by a sponsor or originator by transferring assets to the SPV. Its sole purpose is to collect principal and interest cash flows from the underlying assets and pass them to the owners of the various tranches.

${ }^{28} \mathrm{SIVs}$ also issue medium-term notes and capital notes, which are generally ranked lowest in order of repayment and have a longer maturity. Sponsoring banks usually invest in capital notes.

${ }^{29}$ For details of the issues discussed here, see FitchRatings (September 12, 2007), BearStearns (August 9, 2007), IMF (October, 2007), IMF (December, 2007), Gorton (2008), Brunnermeier (2009), and Coval, Jurek and Stafford (2009).
} 
the FI. In addition, liquidity support to conduits or SIVs by sponsoring banks is regarded as the liquidity support of the FI. The inability of the market to identify and quantify precisely what losses lie in the MBSs and CDOs or their original assets corresponds to the information structure of the participating agents in our model. Because the potential profitability of the original assets such as subprime (or alternative-A) mortgages and credit card, auto, and buyout loans is sufficiently high, Proposition 3 (or Lemma 6) suggests that the equilibrium is located in the UFE region in Figure 2. Hence, the inefficient liquidity support and overinvestment problems can arise because sponsoring banks with deteriorated assets are expected to continue to invest in MBSs and CDOs or the original assets. This tendency is strengthened further if the liquidation value of MBSs and CDOs decreases as a result of the decline in the original assets. Moreover, this kind of equilibrium is more likely to occur, as the repayment likelihood of original asset loan borrowers is ex ante expected to be higher. In recent years, two institutional and structural features have also played an important role in the likelihood of the occurrence of this kind equilibrium. One is regulatory and ratings arbitrage, whereby sponsoring banks took most of their risky loans off their balance sheets so as not to hold costly capital against them. ${ }^{30}$ The other is a low-interest-rate environment, in which securitized and structured products enjoyed popularity among portfolio managers because many were 'searching for yield'. These features further strengthened the occurrence of equilibrium with inefficient liquidity support and overinvestment.

\subsection{Securitization by federal mortgage agencies.-}

The federal mortgage agencies (Fannie Mae and Freddie Mac) issue MBSs (such as the Gold Participate Certificate by Freddie Mac). They also act as sponsors for structured mortgage products (such as the real estate mortgage investment conduits program by Freddie Mac), referred to as collateralized mortgage obligations (CMOs), in which cash flows from the underlying assets are allocated to tranches of varying maturities, coupons, and payment priorities. Because CMOs are backed by MBSs guaranteed by these agencies, the securitization involves a liquidity problem similar to that in Section 5.1.

\section{Extensions and Robustness Checks}

\subsection{Optimality of the short-term debt.-}

\footnotetext{
${ }^{30}$ For the effect of existing regulations on the quality of mortgage loan originations, see Keys et al. (2009).
} 
If the FI can choose to offer an initial security that prevents the UI from withdrawing at time 1, the FI need not consider the incentive compatibility constraint for the UI with respect to whether the UI withdraws its funds at time 1. Even in this case, we can show that this initial security is dominated by a security that allows the UI to withdraw at time 1. The reason is that if the UI cannot withdraw until time 2 , Lemma 1 suggests that the FI always chooses to continue. Thus, we can show that the ex ante expected payoff of the FI is equal to $\widehat{\Pi}_{F 0}$ given in Lemma 5 or 9 , regardless of whether the initial security is backed by the cash flow of $A_{I}$ alone or of both $A_{I}$ and $A_{O}$ (see the Appendix). ${ }^{31}$ Hence, all of the propositions and lemmas in Sections 3 and 4 continue to hold.

\subsection{Optimality of the new security backed by both $A_{I}$ and $A_{O}$ at time 1 .-}

Even if the FI issues a new security backed by $A_{O}$ alone at time 1, it follows from Assumption 4 that the possibility of bankruptcy of the FI $m$ or FI $\ell$ in the new security is exactly the same as in the new security issued at time 1 and backed by $A_{I}$ and $A_{O}$. In addition, in both new securities, the bankruptcy case is equivalent to the case where no cash flow is generated. Hence, we can exclude the new security backed by $A_{O}$ alone without loss of generality. However, the FI may issue a new security backed by $A_{I}$ alone at time 1, just like the initial security. This type of new security increases the likelihood of default of the FI $\ell$ more than that of the FIm. In the pooling case, this change increases the expected payoff of the FI $\ell$ but decreases that of the FIm. In the separating case, this change tightens the incentive compatibility constraint for the FI $\ell$ and reduces the expected payoff of the FIm, although it does not affect the expected payoff of the FI $\ell{ }^{32}$ These arguments show that outside investors with D1 beliefs consider the FI to be the FI $\ell$ if the FI offers the new security backed by $A_{I}$ alone at time 1 in the pooling equilibrium, and that the FI $m$ has no incentive to offer the new security backed by $A_{I}$ alone at time 1 in the separating equilibrium.

\footnotetext{
${ }^{31}$ In fact, if $\frac{1}{\sigma_{\ell}}>\frac{R}{I} \geq \max \left(\phi(\mathcal{L}), \Re_{2}\right)$, the expected payoff of the FI at time 0 under the long-term debt is the same as that under the short-term debt. However, there are several reasons why the short-term debt has additional advantages. First, the UI prefers the short-term maturity. For example, if the UI is faced with liquidity needs when $s=b$, it may have to reserve funds at time 1 . Then, if the UI can satisfy its liquidity needs using the withdrawn funds, the FI can reduce compensation for the UI's cash hoarding costs by issuing short-term debt. Second, in this range of $\frac{R}{T}$, the face value of the long-term debt must be larger than that of the short-term debt because the UI can always recover the investment funds under the short-term debt. If $R$ is not large enough to cover the face value of the long-term debt, this would be an additional advantage of the short-term security. Finally, several studies suggest the role of the short-term debt in reassuring investors in a bank that they will not be too badly expropriated by bank managers (see Calomiris and Kahn (1991), Flannery (1994), and Diamond and Rajan (2001)).

${ }^{32}$ Note that the effect on the FI's borrowing cost for the new security is different from that for the initial security because the FI knows its own $A_{I}$.
} 


\subsection{Nonoptimality of the FI repurchasing the initial security at time 1.}

Without loss of generality, we focus on the case of $s=b$. We also restrict ourselves to the case of informed finance because under Assumption 2, the FI has no incentive to buy back the security at time 1 in order to liquidate $A_{I}$ if it has no private information on $q$.

If at time 1 the FI repurchases the initial security backed by $A_{I}$, the UI must at least be assured of the interim expected payoff attained when withdrawing. Otherwise, the UI rejects the repurchase offer and withdraws or continues. Hence, given the FI's strategy derived in Lemma 4 , the minimum payment to be paid to the UI for repurchase is: (i) if $\frac{1}{\sigma_{\ell}}>\frac{R}{I} \geq$ $\psi(\mathcal{L})$, it is $I$; and (ii) if $\psi(\mathcal{L})>\frac{R}{I}>\frac{1}{\sigma_{m}}$, it is $\delta I+(1-\delta) L$. In both cases, the FI $\ell$ has no incentive to repurchase the security at time 1 because $I>\delta I+(1-\delta) L>L>\sigma_{\ell} R$. With the expectation that only the FI $m$ will repurchase the security, the UI requires at least $I$. Whichever case occurs, neither the FIm nor the FI $\ell$ has any positive incentive to repurchase the security.

\section{Conclusion}

This paper explored the role of nonrecourse financing of securitization by an FI. In equilibrium, even though the FI may provide liquidity support afterward, a security with a nonrecourse property backed by a particular FI asset is ex ante preferred to any security backed by all of its assets (such as deposit claims), because the nonrecourse security makes liquidation of the original asset more attractive for an FI that knows that the original assets are bad. Furthermore, it is also optimal for the FI to offer a security that induces the early withdrawal of uninformed investors when uninformed investors learn that future economic conditions will be bad. However, uninformed finance, inefficient liquidity support, and overinvestment are more likely to arise if the profitability of the asset at the high revenue state increases or if the liquidation value of the asset decreases relative to the amount funded by outside initial uninformed investors or if the likelihood of success with the asset investment increases. The theoretical results have empirical implications for recent problems with securitized and structured finance in the United States and Europe.

However, the application of our model is not necessarily restricted to the issue of securitization. For example, in the Japanese main bank system following the end of the 1990s, non-main banks withdrew their funds early when they suspected that the borrowing firms were likely to become financially distressed. Whether the withdrawal of non-main banks can provide a threat that disciplines the misbehavior of the main bank is an empirical issue. 


\section{Appendix}

Proof of Lemma 2: We first examine the pooling strategy of the FI. ${ }^{33}$

Lemma A1: Under the pooling strategy, both the FIm and the FIl prefer to continue to invest in $A_{I}$ if and only if $\frac{R}{I} \geq \Re_{1}$. The repayment level, $Y^{P}$, is $Y^{P}=\frac{I}{\sigma_{b}+\left(1-\sigma_{b}\right) \sigma_{Z}}$.

Proof of Lemma A1: In this case, both the FIm and the FI $\ell$ use the same strategy. We subsequently solve for the equilibrium $Y^{P}$ by assuming that $Z \geq Y^{P}$ holds, and then we verify that the equilibrium value of $Y^{P}$ really satisfies $Z \geq Y^{P}$. Suppose that both the FIm and the FI $\ell$ continue. If $Z \geq Y^{P}$, the interim expected payoff obtained by the FI with $q$ (or by outside investors), $\Pi_{F 2}^{P}(q)$ (or $\left.\Pi_{O 2}^{P}\right)$, under the pooling strategy is $\Pi_{F 2}^{P}(q)=\sigma_{q}\left(R-Y^{P}\right.$ $+Z)+\left(1-\sigma_{q}\right) \sigma_{Z}\left(Z-Y^{P}\right)\left(\right.$ or $\left.\Pi_{O 2}^{P}=\sum_{q} \delta_{q}\left[\sigma_{q}+\left(1-\sigma_{q}\right) \sigma_{Z}\right] Y^{P}\right)$, where $\delta_{m}=\delta$ and $\delta_{\ell}$ $=1-\delta$. Note that under the pooling strategy, outside investors expect that the repayment probability is $\sum_{q} \delta_{q}\left[\sigma_{q}+\left(1-\sigma_{q}\right) \sigma_{Z}\right]$. It follows from the participation constraint for outside investors that $Y^{P}$ is determined by $Y^{P}=\frac{I}{\sum_{q} \delta_{q}\left[\sigma_{q}+\left(1-\sigma_{q}\right) \sigma_{Z}\right]}=\frac{I}{\sigma_{b}+\left(1-\sigma_{b}\right) \sigma_{Z}}$. Under Assumption 4, this $Y^{P}$ satisfies the condition $Z \geq Y^{P}$. Substituting $Y^{P}$ into $\Pi_{F 2}^{P}(q)$ yields

$$
\Pi_{F 2}^{P}(q)=\sigma_{q} R+\left[\sigma_{q}+\left(1-\sigma_{q}\right) \sigma_{Z}\right] Z-\frac{\sigma_{q}+\left(1-\sigma_{q}\right) \sigma_{Z}}{\sigma_{b}+\left(1-\sigma_{b}\right) \sigma_{Z}} I
$$

Now, suppose that the FI with $q$ liquidates $A_{I}$. Because the FI's claim is junior, we have

$$
\Pi_{F 2}^{P}(q)=\left[\sigma_{q}+\left(1-\sigma_{q}\right) \sigma_{Z}\right] Z
$$

Comparing (A1) and (A2), we show that the FI with $q$ prefers to continue as long as $R \geq$ $\frac{\sigma_{q}+\left(1-\sigma_{q}\right) \sigma_{Z}}{\sigma_{q}\left[\sigma_{b}+\left(1-\sigma_{b}\right) \sigma_{Z}\right]} I \equiv \psi\left(\sigma_{q}\right)$. Because $\sigma_{m}>\sigma_{\ell}$, we obtain $\psi\left(\sigma_{\ell}\right)>\psi\left(\sigma_{m}\right)$. As both the FI $m$ and the FI $\ell$ make the same choice under the pooling strategy, they prefer to continue after the withdrawal of the UI under the pooling strategy if and only if $\frac{R}{I} \geq \Re_{1}$. $\|$

Because the situation where both the FIm and the FI $\ell$ prefer to liquidate $A_{I}$ after the UI withdraws is the residual case in which neither the pooling strategy with continuation nor the separating strategy is chosen, we proceed to discuss the separating strategy. Indeed, outside investors demand at least $\frac{I}{\sigma_{\ell}+\left(1-\sigma_{\ell}\right) \sigma_{Z}}$ of the FI $\ell$ if they know that the FI is the FI $\ell$. However, the expected payoff of the $\mathrm{FI} \ell$ is then larger in liquidation than in continuation

\footnotetext{
${ }^{33}$ We assume that the FI chooses to continue if it has no preference between continuing and liquidating.
} 
because $\left[\sigma_{\ell}+\left(1-\sigma_{\ell}\right) \sigma_{Z}\right] Z>\sigma_{\ell}\left[R+Z-\frac{I}{\sigma_{\ell}+\left(1-\sigma_{\ell}\right) \sigma_{Z}}\right]+\left(1-\sigma_{\ell}\right) \sigma_{Z}\left[Z-\frac{I}{\sigma_{\ell}+\left(1-\sigma_{\ell}\right) \sigma_{Z}}\right]$ under Assumption 1. This implies that if the separating strategy is possible, the FIm continues while the FI $\ell$ liquidates. Thus, outside investors can perfectly infer the innate value of their debt claim from the decision by the FI to continue or liquidate under the separating strategy. Now, we have the following lemma.

Lemma A2: Under the separating strategy, the FIm continues to invest in $A_{I}$ while the FIl liquidates $A_{I}$. The repayment schedule is given by $Y_{R+Z}^{m}=Y_{Z}^{m}=Y^{m}$, where $Y^{m}$ $=\frac{\sigma_{\ell} R}{\sigma_{\ell}+\left(1-\sigma_{\ell}\right) \sigma_{Z}}\left(>\frac{I}{\sigma_{m}+\left(1-\sigma_{m}\right) \sigma_{Z}}\right)$ if $\frac{R}{I} \geq \Re_{2}$; and $Y^{m}=\frac{I}{\sigma_{m}+\left(1-\sigma_{m}\right) \sigma_{Z}}$ if $\frac{R}{I}<\Re_{2}$. No other separating strategy is viable.

Proof of Lemma A2: We only need to prove the case where the FIm continues while the FI $\ell$ liquidates. We first show that $Y_{R+Z}^{m}=Y_{Z}^{m}=Y^{m}$ if $Z \geq Y^{m}$. Indeed, the most effective security design for preventing the FI $\ell$ from pretending to be the FIm is that $Y_{R+Z}^{m}=Y_{Z}^{m}=$ $Y^{m}$, because it follows from the monotonicity assumption that the expected repayment of the $\mathrm{FI} \ell$ is then largest if $Z \geq Y^{m}$. We will thus solve for the equilibrium $Y^{m}$ by assuming $Z$ $\geq Y^{m}$, and we then verify that the equilibrium value of $Y^{m}$ satisfies $Z \geq Y^{m}$.

If $Z \geq Y^{m}$ holds, the expected payoff obtained by the FIm (or by outside investors), $\Pi_{F 2}^{S}\left(m, Y^{m}\right)\left(\right.$ or $\left.\Pi_{O 2}^{S}\left(Y^{m}\right)\right)$, is $\Pi_{F 2}^{S}\left(m, Y^{m}\right)=\sigma_{m}\left(R-Y^{m}+Z\right)+\left(1-\sigma_{m}\right) \sigma_{Z}\left(Z-Y^{m}\right)$ (or $\left.\Pi_{O 2}^{S}\left(Y^{m}\right)=\left[\sigma_{m}+\left(1-\sigma_{m}\right) \sigma_{Z}\right] Y^{m}\right)$. Note that under the separating strategy, outside investors expect that the repayment probability is $\sigma_{m}+\left(1-\sigma_{m}\right) \sigma_{Z}$. On the other hand, if the FI $\ell$ mimics the strategy of the FIm, the expected payoff of the FI $\ell$ is

$$
\Pi_{F 2}^{S}\left(\ell, Y^{m}\right)=\sigma_{\ell}\left(R-Y^{m}+Z\right)+\left(1-\sigma_{\ell}\right) \sigma_{Z}\left(Z-Y^{m}\right)
$$

The self-selection constraint of the FI $\ell$ and the participation constraint of the outside investors are now characterized by

$$
\begin{gathered}
{\left[\sigma_{\ell}+\left(1-\sigma_{\ell}\right) \sigma_{Z}\right] Z \geq \sigma_{\ell}\left(R-Y^{m}+Z\right)+\left(1-\sigma_{\ell}\right) \sigma_{Z}\left(Z-Y^{m}\right)} \\
{\left[\sigma_{m}+\left(1-\sigma_{m}\right) \sigma_{Z}\right] Y^{m} \geq I .}
\end{gathered}
$$

Note that the FI $\ell$ obtains $\left[\sigma_{\ell}+\left(1-\sigma_{\ell}\right) \sigma_{Z}\right] Z$ if it does not mimic the FIm; that is, it liquidates $A_{I}$. Solving (A4) and (A5) with respect to $Y^{m}$ yields $Y^{m} \geq \max \left[\frac{\sigma_{\ell} R}{\sigma_{\ell}+\left(1-\sigma_{\ell}\right) \sigma_{Z}}, \frac{I}{\sigma_{m}+\left(1-\sigma_{m}\right) \sigma_{Z}}\right]$. If $Z \geq Y^{m}$, then $\Pi_{F 2}^{S}\left(m, Y^{m}\right)$ is maximized if the FIm offers the following security: $Y^{m}=$ $\frac{\sigma_{\ell} R}{\sigma_{\ell}+\left(1-\sigma_{\ell}\right) \sigma_{Z}}$ if $\frac{R}{I} \geq \Re_{2}$, and $Y^{m}=\frac{I}{\sigma_{m}+\left(1-\sigma_{m}\right) \sigma_{Z}}$ if $\frac{R}{I}<\Re_{2}$. Note that the security satisfies $Z$ 
$\geq Y^{m}$ under Assumption 4. Substituting $Y^{m}$ into $\Pi_{F 2}^{S}\left(m, Y^{m}\right)$, we obtain

$$
\begin{gathered}
\Pi_{F 2}^{S}(m)=\sigma_{m} R+\left[\sigma_{m}+\left(1-\sigma_{m}\right) \sigma_{Z}\right] Z-\frac{\sigma_{m}+\left(1-\sigma_{m}\right) \sigma_{Z}}{\sigma_{\ell}+\left(1-\sigma_{\ell}\right) \sigma_{Z}} \sigma_{\ell} R \quad \text { if } \frac{R}{I} \geq \Re_{2}, \\
\Pi_{F 2}^{S}(m)=\sigma_{m} R+\left[\sigma_{m}+\left(1-\sigma_{m}\right) \sigma_{Z}\right] Z-I \quad \text { if } \frac{R}{I}<\Re_{2} .
\end{gathered}
$$

The remaining problem is to show that the separating equilibrium is possible; that is, the FIm prefers continuing rather than liquidating. Indeed, it follows from Assumption 1 and $\sigma_{m}>\sigma_{\ell}$ that $\Pi_{F 2}^{S}(m) \geq\left[\sigma_{m}+\left(1-\sigma_{m}\right) \sigma_{Z}\right] Z$, regardless of whether $\frac{R}{I} \geq \Re_{2}$ or $\frac{R}{I}<$ $\Re_{2}$. Because the expected payoff obtained by the FI $m$ in liquidation is equal to $\left[\sigma_{m}+(1-\right.$ $\left.\left.\sigma_{m}\right) \sigma_{Z}\right] Z$, the FIm prefers continuing rather than liquidating. $\|$

Now, let us note that $\Re_{1}>\Re_{2}$ because of $\sigma_{m}>\sigma_{b}$. It follows from the proofs of Lemmas A1 and A2 that the expected payoff of the FI with $q$ under the optimal pooling strategy, $\Pi_{F 2}^{P *}(q)$, is given by (A1) if and only if $\frac{R}{I} \geq \Re_{1}$, while the expected payoff of the FI $m$ under the optimal separating strategy, $\Pi_{F 2}^{S *}(m)$, is given by (A6) or (A7) according to $\frac{R}{I} \geq \Re_{2}$ or $\frac{R}{I}<\Re_{2}$. Furthermore, the expected payoff of the FI $\ell$ under the optimal separating strategy, $\Pi_{F 2}^{S *}(\ell)$, equals $\left[\sigma_{\ell}+\left(1-\sigma_{\ell}\right) \sigma_{Z}\right] Z$.

Suppose that $\frac{R}{I} \geq \Re_{1}$. Then, it follows from (A1) and (A6) with the range $\frac{R}{I}$ that $\Pi_{F 2}^{P *}(m)$ $\geq \Pi_{F 2}^{S *}(m)$ and $\Pi_{F 2}^{P *}(\ell)>\Pi_{F 2}^{S *}(\ell)$. Thus, in this range $\frac{R}{I}$, the optimal pooling strategy given in Lemma A1 dominates the optimal separating strategy given in Lemma A2.

If $\Re_{1}>\frac{R}{I}$, the pooling strategy given in Lemma A1 is infeasible. Hence, we must compare the optimal separating strategy given in Lemma A2 and the pooling strategy in which both the FI $m$ and the FI $\ell$ prefer to liquidate after the UI withdraws. Suppose that the liquidation strategy is switched to the continuation strategy with $Y^{m}=\frac{\sigma_{\ell} R}{\sigma_{\ell}+\left(1-\sigma_{\ell}\right) \sigma_{Z}}$ when $\frac{R}{I} \geq \Re_{2}$ or with $Y^{m}=\frac{I}{\sigma_{m}+\left(1-\sigma_{m}\right) \sigma_{Z}}$ when $\frac{R}{I}<\Re_{2}$. Then, the expected payoff of the FI $m$ increases, because it follows from (A6) and (A7) with $\sigma_{m}>\sigma_{\ell}$ and $\sigma_{m} R>I$ that $\Pi_{F 2}^{S *}(m)>\left[\sigma_{m}+(1\right.$ $\left.\left.-\sigma_{m}\right) \sigma_{Z}\right] Z$, while the expected payoff of the FI $\ell$ does not increase, because it follows from (A3) and the range of $\frac{R}{I}$ that $\left[\sigma_{\ell}+\left(1-\sigma_{\ell}\right) \sigma_{Z}\right] Z \geq \Pi_{F 2}^{S}\left(\ell, Y^{m}\right)$. Now, the FI $m$ has more incentive to switch to the continuation strategy defined above than does the FI $\ell$. Hence, if the FI chooses the continuation strategy with $Y^{m}=\frac{\sigma_{\ell} R}{\sigma_{\ell}+\left(1-\sigma_{\ell}\right) \sigma_{Z}}$ or $Y^{m}=\frac{I}{\sigma_{m}+\left(1-\sigma_{m}\right) \sigma_{Z}}$ according to $\frac{R}{I} \geq \Re_{2}$ or $\frac{R}{I}<\Re_{2}$, outside investors with D1 beliefs think that the FI is the FI $m$. The pooling strategy with liquidation is thus pruned from the game.

Proof of Lemma 3: (i) If $\frac{R}{I} \geq \Re_{1}$, Lemma 2 implies that both the FIm and the FI $\ell$ 
continue after the withdrawal of the UI. If the UI withdraws, it always receives repayment $I$. If the UI continues, the interim expected payoff is $\sigma_{b} X$ because the FI continues while the UI does not know the type of FI. Thus, the UI continues if and only if $\sigma_{b} X \geq I$.

(ii) If $\Re_{1}>\frac{R}{I}$, Lemma 2 shows that after the withdrawal of the UI, the FI $m$ continues, whereas the FI $\ell$ liquidates. Then, if the UI withdraws, it receives repayment $I$ when the FI is the FIm, while it receives repayment $L$ under the assumed priority rule when the FI is the FI $\ell$. Thus, the UI continues if and only if $\sigma_{b} X \geq \delta I+(1-\delta) L$.

Proof of Lemma 4: To prove Lemma 4, we prove the following lemma.

Lemma A3: (i) Suppose that $\frac{1}{\sigma_{\ell}}>\frac{R}{I} \geq \Re_{1}$. The optimal strategies of the FI and the UI are the same as those of Lemma $4(i)$. The ex ante expected payoff of the FI, $\Pi_{F 0}^{*}$, is $\Pi_{F 0}^{*}=$ $\widehat{\Pi}_{F 0} \equiv\left[p+(1-p) \sigma_{b}\right] R+\omega Z-\bar{I}$, where $\omega \equiv p+(1-p)\left[\sigma_{b}+\left(1-\sigma_{b}\right) \sigma_{Z}\right]$.

(ii) Suppose that $\Re_{1}>\frac{R}{I} \geq \Re_{2}$. (a) If $\Re_{1}>\frac{R}{I} \geq \psi(\mathcal{L})$, the optimal strategies of the $F I$ and the UI are the same as those of Lemma $4(i i)$; and $\Pi_{F 0}^{*}=\left[p+(1-p) \sigma_{b}\right] R+\omega Z-$ $\frac{\delta\left[p+(1-p) \sigma_{b}\right]-\sigma_{b}}{\sigma_{b}} I-\frac{(1-\delta)\left[p+(1-p) \sigma_{b}\right]}{\sigma_{b}} L-\bar{I}$. (b) If $\psi(\mathcal{L})>\frac{R}{I} \geq \Re_{2}$, the optimal strategies of the FI and the UI are the same as those of Lemma $4($ iii $)$; and $\Pi_{F 0}^{*}=\left[p+\frac{(1-p) \delta\left(\sigma_{m}-\sigma_{\ell}\right) \sigma_{Z}}{\sigma_{\ell}+\left(1-\sigma_{\ell}\right) \sigma_{Z}}\right] R+$ $(1-p)(1-\delta) L+\omega Z+(1-p) \delta I-\bar{I}$.

(iii) Suppose that $\Re_{2}>\frac{R}{I}>\frac{1}{\sigma_{m}}$. The optimal strategies of the FI and the UI are the same as those of Lemma 4 (iii); and $\Pi_{F 0}^{*}=\left[p+(1-p) \delta \sigma_{m}\right] R+(1-p)(1-\delta) L+\omega Z-\bar{I}$.

Proof of Lemma A3: (i) In this case, it follows from Lemma 3(i) that the UI continues (or withdraws) at $s=b$ if $\sigma_{b} X \geq I\left(\sigma_{b} X<I\right)$, and it follows from Lemma 1 (or 2(i)) that both the FI $m$ and the FI $\ell$ continue after the UI decides to continue (or withdraw). Hence, if we impose the condition $\sigma_{b} X \geq I$, the security design problem of the FI at time 0 is

$$
\max _{X} \Pi_{F 0}=\max _{X}\left[p+(1-p) \sigma_{b}\right](R-X+Z)+(1-p)\left(1-\sigma_{b}\right) \sigma_{Z} Z-(\bar{I}-I),
$$

subject to the following: (i) (IRU1), $\left[p+(1-p) \sigma_{b}\right] X \geq I$; (ii) (ICU1), $\sigma_{b} X \geq I$; and (iii) (NR), $R \geq X \geq 0$. The objective function represents the ex ante expected payoff of the FI. As the FI continues until time 2 and need not finance $I$ at time 1 in this case, it receives $R$ $+Z$ from the cash flows of $A_{I}$ and $A_{O}$ but repays $X$ with probability $p+(1-p) \sigma_{b}$, and it receives $Z$ from the cash flow of $A_{O}$ with probability $(1-p)\left(1-\sigma_{b}\right) \sigma_{Z}$. Note that the FI does not know $q$ at time 0 , and must contribute $\bar{I}-I$. (IRU1) is the individual rationality constraint for the UI. (ICU1) ensures that the UI continues at $s=b$. (NR) is the nonrecourse constraint of each agent's claim. 
If we impose the condition $\sigma_{b} X<I$, then the FI's problem is

$$
\begin{aligned}
\max _{X} \Pi_{F 0}= & \max _{X} p(R-X+Z)+(1-p)\left\{\sigma_{b}\left[R+Z-\frac{I}{\sigma_{b}+\left(1-\sigma_{b}\right) \sigma_{Z}}\right]\right. \\
& \left.+\left(1-\sigma_{b}\right) \sigma_{Z}\left[Z-\frac{I}{\sigma_{b}+\left(1-\sigma_{b}\right) \sigma_{Z}}\right]\right\}-(\bar{I}-I),
\end{aligned}
$$

subject to the following: (i) (IRU2), $p X+(1-p) I \geq I$; (ii) (ICU2), $\sigma_{b} X<I$; and (iii) (NR). The objective function represents the ex ante expected payoff of the FI. As the FI continues until time 2 but must finance $I$ at time 1 when $s=b$, it receives $R+Z$ from the cash flows of $A_{I}$ and $A_{O}$ but repays $X$ with probability $p$, receives $R+Z$ from the cash flows of $A_{I}$ and $A_{O}$ but repays $\frac{I}{\sigma_{b}+\left(1-\sigma_{b}\right) \sigma_{Z}}$ with probability $(1-p) \sigma_{b}$, and receives $Z$ from the cash flow of $A_{O}$ but repays $\frac{I}{\sigma_{b}+\left(1-\sigma_{b}\right) \sigma_{Z}}$ with probability $(1-p)\left(1-\sigma_{b}\right) \sigma_{Z}$. Note that the repayment to outside investors in this case is given by Lemma 2(i). (IRU2) is the individual rationality constraint for the UI. (ICU2) ensures that the UI withdraws at $s=b$.

Now, comparing the optimal value of problem (P1) with that of problem (P2) yields the optimal initial security in the case of $\frac{1}{\sigma_{\ell}}>\frac{R}{I} \geq \Re_{1}$. We begin by analyzing problem (P1). Rearranging (IRU1) and (ICU1), we have $X \geq \frac{I}{p+(1-p) \sigma_{b}}$ and $X \geq \frac{I}{\sigma_{b}}$. It follows from $\frac{I}{p+(1-p) \sigma_{b}}<\frac{I}{\sigma_{b}}$ that (ICU1) is more likely to bind than (IRU1). Because $\Pi_{F 0}$ decreases in $X$, $\Pi_{F 0}$ is maximized by $X^{c}$, which is the minimal value of $X$ that satisfies both (ICU1) and $(\mathrm{NR})$; that is, $X^{c}=\frac{I}{\sigma_{b}}$. Note that $X^{c}$ satisfies (NR) because $\frac{R}{I} \geq \Re_{1}\left(>\frac{1}{\sigma_{b}}\right.$ under $\left.\sigma_{m}>\sigma_{\ell}\right)$. Substituting this $X^{c}$ into $\Pi_{F 0}$ of $(\mathrm{P} 1)$, we obtain

$$
\Pi_{F 0}^{c}=\left[p+(1-p) \sigma_{b}\right] R+\omega Z-\frac{p\left(1-\sigma_{b}\right)}{\sigma_{b}} I-\bar{I}
$$

We next deal with problem (P2). Rearranging (IRU2) and (ICU2), we have $I \leq X<\frac{I}{\sigma_{b}}$, which is nonempty. Because $\Pi_{F 0}$ decreases in $X, \Pi_{F 0}$ is minimized by $X^{w}$; that is, $X^{w}=I$. Note that $X^{w}$ satisfies (NR). Substituting this $X^{w}$ into $\Pi_{F 0}$ of (P2), we have

$$
\Pi_{F 0}^{w}=\widehat{\Pi}_{F 0} \equiv\left[p+(1-p) \sigma_{b}\right] R+\omega Z-\bar{I}
$$

Comparing (A8) and (A9), we obtain the optimal solution given in Lemma A3(i).

(ii) In this case, it follows from Lemma 3(ii) that the UI continues (or withdraws) at $s=$ $b$ if $\sigma_{b} X \geq \delta I+(1-\delta) L\left(\right.$ or $\left.\sigma_{b} X<\delta I+(1-\delta) L\right)$, and it follows from Lemma 1 (or 
2(ii)) that both the FIm and the FI $\ell$ continue after the continuation of the UI (or the FIm continues but the FI $\ell$ liquidates after the withdrawal of the UI). If we impose the condition that $\sigma_{b} X \geq \delta I+(1-\delta) L$, the security design problem of the FI at time 0 is characterized by

$$
\max _{X} \Pi_{F 0} \text { given by }(\mathrm{P} 1),
$$

subject to the following: (i) (IRU1); (ii) (ICU3), $\sigma_{b} X \geq \delta I+(1-\delta) L$; and (iii) (NR). Note that (ICU3) ensures that the UI continues at $s=b$.

By contrast, if the condition that $\sigma_{b} X<\delta I+(1-\delta) L$ is imposed, the FI's problem is

$$
\begin{gathered}
\max _{X} \Pi_{F 0}=\max _{X} p(R-X+Z)+(1-p)\left\{\delta \sigma_{m}\left[R+Z-\frac{\sigma_{\ell} R}{\sigma_{\ell}+\left(1-\sigma_{\ell}\right) \sigma_{Z}}\right]\right. \\
\left.+\delta\left(1-\sigma_{m}\right) \sigma_{Z}\left[Z-\frac{\sigma_{\ell} R}{\sigma_{\ell}+\left(1-\sigma_{\ell}\right) \sigma_{Z}}\right]+(1-\delta)\left[\sigma_{\ell}+\left(1-\sigma_{\ell}\right) \sigma_{Z}\right] Z\right\}-(\bar{I}-I),
\end{gathered}
$$

subject to the following: (i) (IRU4), $p X+(1-p)[\delta I+(1-\delta) L] \geq I$; (ii) (ICU4), $\sigma_{b} X<$ $\delta I+(1-\delta) L$; and (iii) (NR). The objective function represents the ex ante expected payoff of the FI. As the FIm (FI $\ell$ ) continues (liquidates) after the UI withdraws, the FI receives $R$ $+Z$ from the cash flows of $A_{I}$ and $A_{O}$ but repays $X$ with probability $p$, receives $R+Z$ from the cash flows of $A_{I}$ and $A_{O}$ but repays $\frac{\sigma_{\ell} R}{\sigma_{\ell}+\left(1-\sigma_{\ell}\right) \sigma_{Z}}$ with probability $(1-p) \delta \sigma_{m}$, receives $Z$ from the cash flow of $A_{O}$ but repays $\frac{\sigma_{\ell} R}{\sigma_{\ell}+\left(1-\sigma_{\ell}\right) \sigma_{Z}}$ with probability $(1-p) \delta\left(1-\sigma_{m}\right) \sigma_{Z}$, and only receives $Z$ with probability $(1-p)(1-\delta)\left[\sigma_{\ell}+\left(1-\sigma_{\ell}\right) \sigma_{Z}\right]$ (see Table 1$)$. Note that the repayment to outside investors is given by Lemma 2(ii). (IRU4) is the individual rationality constraint for the UI. (ICU4) ensures that the UI withdraws at $s=b$.

Comparing the optimal value of problem (P3) with problem (P4) leads to the optimal security in the case of $\Re_{1}>\frac{R}{I} \geq \Re_{2}$. We begin by solving problem (P3). Using a procedure similar to solving problem $(\mathrm{P} 1)$, we have $X^{c}=\frac{\delta I+(1-\delta) L}{\sigma_{b}}$ and

$$
\Pi_{F 0}^{c}=\left[p+(1-p) \sigma_{b}\right] R+\omega Z-\frac{p+(1-p) \sigma_{b}}{\sigma_{b}}[\delta I+(1-\delta) L]-(\bar{I}-I),
$$

if only (ICU3) binds, that is, if

$$
\frac{\delta\left[p+(1-p) \sigma_{b}\right]-\sigma_{b}}{p+(1-p) \sigma_{b}}+(1-\delta) \mathcal{L}>0
$$


Note that $X^{c}<R$ for $\frac{R}{I} \geq \Re_{2}$ (> $\frac{1}{\sigma_{b}}$ under Assumption 5(ii)), and that (A11) is satisfied under Assumption 5(i) because $\mathcal{L}>\sigma_{\ell} \frac{R}{I}>\frac{\sigma_{\ell}}{\sigma_{m}}$ from Assumption 1. We next proceed to solve problem (P4). Again, using a procedure similar to solving problem (P2), we obtain $X^{w}$ $=\frac{[1-(1-p) \delta] I-(1-p)(1-\delta) L}{p}$ if only (IRU4) binds, that is, if (A11) is satisfied. Otherwise, there is no solution. Note that $X^{w}<R$ and (A11) are satisfied for $\frac{R}{I}>\frac{1}{\sigma_{m}}$ and $\mathcal{L}>\frac{\sigma_{\ell}}{\sigma_{m}}$ under Assumptions 1 and 5(i). Substituting $X^{w}$ into $\Pi_{F O}$ of $(\mathrm{P} 4)$, we see

$$
\Pi_{F 0}^{w}=\left[p+\frac{(1-p) \delta\left(\sigma_{m}-\sigma_{\ell}\right) \sigma_{Z}}{\sigma_{\ell}+\left(1-\sigma_{\ell}\right) \sigma_{Z}}\right] R+(1-p)(1-\delta) L+\omega Z+(1-p) \delta I-\bar{I}
$$

Comparing (A10) and (A12) yields the optimal solution when $\Re_{1}>\frac{R}{I} \geq \Re_{2}$ : it is given by Lemma A3(ii)(a) if $\Re_{1}>\frac{R}{I} \geq \psi(\mathcal{L})$, and is given by Lemma A3(ii)(b) if $\psi(\mathcal{L})>\frac{R}{I} \geq \Re_{2}$.

(iii) In this case, the decision criterion of the UI continuing or withdrawing is the same as the case when $\Re_{1}>\frac{R}{I} \geq \Re_{2}$. Hence, we examine the two situations: $\sigma_{b} X \geq \delta I+(1-\delta) L$ or $\sigma_{b} X<\delta I+(1-\delta) L$. In the former, the FI's problem is the same as in (P3) because the UI continues. In the latter, where the UI withdraws, the FI's problem is represented by

$$
\begin{gathered}
\max _{X} \Pi_{F 0}=\max _{X} p(R-X+Z)+(1-p)\left\{\delta \sigma_{m}\left[R+Z-\frac{I}{\sigma_{m}+\left(1-\sigma_{m}\right) \sigma_{Z}}\right]\right. \\
\left.+\delta\left(1-\sigma_{m}\right) \sigma_{Z}\left[Z-\frac{I}{\sigma_{m}+\left(1-\sigma_{m}\right) \sigma_{Z}}\right]+(1-\delta)\left[\sigma_{\ell}+\left(1-\sigma_{\ell}\right) \sigma_{Z}\right] Z\right\}-(\bar{I}-I),
\end{gathered}
$$

subject to (IRU4), (ICU4), and (NR). Note that the repayment to outside investors is given by Lemma 2(iii).

Comparing the optimal value of problem (P3) with that of problem (P5) yields the optimal initial security when $\Re_{2}>\frac{R}{I}>\frac{1}{\sigma_{m}}$. We solve problem (P5) in a similar way to problem (P4). Under Assumptions 1 and 5(i), we have $X^{w}=\frac{[1-(1-p) \delta] I-(1-p)(1-\delta) L}{p}$ and

$$
\Pi_{F 0}^{w}=\left[p+(1-p) \delta \sigma_{m}\right] R+(1-p)(1-\delta) L+\omega Z-\bar{I}
$$

Comparing (A10) and (A13) gives the optimal solution of Lemma A3(iii) under Assumptions 1 and $5(\mathrm{i})$.

The results of Lemma 4 are immediate from Lemma A3.

Proof of Lemma 5: The UFE is obtained by the same backward induction procedure as 
that of Section 3. We first discuss the optimal decisions of the FI and the UI at time 1. Again, at $s=g$, it is trivial that both the FI and the UI choose to continue. Hence, we focus on the case of $s=b$. Let us first examine the FI's continuation/liquidation decision after the UI withdraws. Then, only the pooling equilibrium needs to be considered because the FI does not know which $q$ is realized. Thus, the repayment level of the FI in the capital market is $\frac{I}{\sigma_{b}+\left(1-\sigma_{b}\right) \sigma_{Z}}$. Because the FI receives nothing if liquidation occurs, the FI continues (liquidates) after the withdrawal of the UI if $\sigma_{b} R-I \geq 0\left(\sigma_{b} R-I<0\right)$. For the FI's continuation/liquidation decision after the UI continues, the result is the same as Lemma 1. Now, let us discuss the continuation/withdrawal decision of the UI at time 1 . Then, the argument of the FI's continuation/liquidation decision stated above leads to the following two cases: (i) $\frac{1}{\sigma_{\ell}}>\frac{R}{I} \geq \frac{1}{\sigma_{b}}$; and (ii) $\frac{1}{\sigma_{b}}>\frac{R}{I}>\frac{1}{\sigma_{m}}$. In the first case, the FI continues after the withdrawal of the UI. Hence, the UI continues (or withdraws) if and only if $\sigma_{b} X \geq I$ (or $\left.\sigma_{b} X<I\right)$. In the second case, the FI liquidates after the withdrawal of the UI. Thus, the UI continues (or withdraws) if and only if $\sigma_{b} X \geq L$ (or $\sigma_{b} X<L$ ).

Now, we proceed to explore the FI's security offer at time 0. Again, we analyze the following two cases: (i) $\frac{1}{\sigma_{\ell}}>\frac{R}{I} \geq \frac{1}{\sigma_{b}}$; and (ii) $\frac{1}{\sigma_{b}}>\frac{R}{I}>\frac{1}{\sigma_{m}}$. Furthermore, for each of these cases, we need to consider both the situations in which: (a) the UI is induced to continue at $s=b$; and (b) the UI is induced to withdraw at $s=b$. Given the optimal decisions of the FI and the UI at time 1 , if $\frac{1}{\sigma_{\ell}}>\frac{R}{I} \geq \frac{1}{\sigma_{b}}$, we solve the same problems as (P1) and (P2) that are in the proof of Lemma 4 , and compare their optimal values of $\Pi_{F 0}$. Then, it follows from Lemma A3(i) that $X^{*}=I$ and $\Pi_{F 0}^{*}=\widehat{\Pi}_{F 0}$. In the case where $\frac{1}{\sigma_{b}}>\frac{R}{I}>\frac{1}{\sigma_{m}}$, if the UI is induced to continue at $s=b$, we solve the same problem as (P1), except that (ICU1) is changed by $\sigma_{b} X \geq L$ because the FI liquidates after the withdrawal of the UI when $\frac{1}{\sigma_{b}}>\frac{R}{I}$ $>\frac{1}{\sigma_{m}}$. Solving this problem in a similar way to problem $(\mathrm{P} 1)$, we can show that $\Pi_{F 0}^{*}=[p$ $\left.+(1-p) \sigma_{b}\right]\left(R-\frac{L}{\sigma_{b}}\right)+\omega Z-(\bar{I}-I)$ if $\mathcal{L}>\frac{\sigma_{b}}{p+(1-p) \sigma_{b}}$; and $\Pi_{F 0}^{*}=\widehat{\Pi}_{F 0}$ if $\mathcal{L} \leq \frac{\sigma_{b}}{p+(1-p) \sigma_{b}}$. On the other hand, if the UI is induced to withdraw at $s=b$, the FI solves the following problem: $\max _{X} \Pi_{F 0} \equiv \max _{X} p(R-X)+\omega Z-(\bar{I}-I)$, subject to $p X+(1-p) L \geq I, \sigma_{b} X$ $<L$ and (NR). Applying a procedure similar to that of Lemma A3(i), we can prove that $\Pi_{F 0}^{*}=p R+(1-p) L+\omega Z-\bar{I}$ if $\mathcal{L}>\frac{\sigma_{b}}{p+(1-p) \sigma_{b}}$; and there is no solution if $\mathcal{L} \leq \frac{\sigma_{b}}{p+(1-p) \sigma_{b}}$. However, in any case of $\frac{1}{\sigma_{b}}>\frac{R}{I}>\frac{1}{\sigma_{m}}$, we can show that $\Pi_{F 0}^{*} \leq \widehat{\Pi}_{F 0}$ under our assumptions. Summarizing these arguments, the result in this lemma is verified.

Proof of Lemma 6: Note that $\Re_{2}>\frac{1}{\sigma_{b}}$ with Assumption 5(ii), and that the FI pays an 
infinitesimally small cost $\epsilon_{I}$ under information acquisition at time 0 . Then, we show that if $\frac{1}{\sigma_{\ell}}>\frac{R}{I} \geq \Re_{1}$, the equilibrium is given by the UFE, which is characterized by Lemma 5(i); and under Assumption 1, if $\Re_{2}>\frac{R}{I}>\frac{1}{\sigma_{m}}$, the equilibrium is given by the IFES, which is featured by Lemma 4(iii) or A3(iii). Now, if $\Re_{1}>\frac{R}{I} \geq \Re_{2}$, comparing $\Pi_{F 0}^{*}$ of Lemma A3(ii)(a) with $\Pi_{F 0}^{*}$ of Lemma $5(\mathrm{i})$ verifies that the former is smaller than the latter under (A11) or Assumption 5(i). Note that $\mathcal{L}>\frac{\sigma_{\ell}}{\sigma_{m}}$ holds in Assumption 1, as proved in Lemma A3(ii). The remaining issue is to check whether $\Pi_{F 0}^{*}$ of Lemma A3(ii)(b) is larger than $\Pi_{F 0}^{*}$ of Lemma $5(\mathrm{i})$. This difference equals $\frac{(1-p) I}{\Re_{1}}\left[\phi(\mathcal{L})-\frac{R}{I}\right]$. Thus, the former is larger (or smaller) than the latter if $\phi(\mathcal{L})>($ or $<) \frac{R}{I}$. Note that $\varphi(\mathcal{L}) \geq \phi(\mathcal{L})$ for $\Re_{1}>\frac{R}{I} \geq \Re_{2}$, because the foregoing argument with the definition of $\varphi(\mathcal{L})$ in Lemma A3 shows that $\left(\Pi_{F 0}^{*}\right.$ of Lemma $5(\mathrm{i})) \geq\left(\Pi_{F 0}^{*}\right.$ of Lemma A3(ii)(a)) $\geq\left(\Pi_{F 0}^{*}\right.$ of Lemma A3(ii)(b)) as long as $\frac{R}{I} \geq$ $\varphi(\mathcal{L})$. However, we cannot determine whether $\phi(\mathcal{L}) \gtreqless \Re_{2}$. Hence, these arguments prove that if $\Re_{1}>\frac{R}{I} \geq \max \left(\phi(\mathcal{L}), \Re_{2}\right)$, the equilibrium is given by the UFE of Lemma $5(\mathrm{i})$; and if $\max \left(\phi(\mathcal{L}), \Re_{2}\right)>\frac{R}{I} \geq \Re_{2}$, the equilibrium is given by the IFES of Lemma 4(iii) or A3(ii)(b).

Proof of Lemma 7: The result of part (i) is evident from Lemma 1. To prove the result of part (ii), we first specify the expected payoff of the FI with $q, \Pi_{F 2}^{P}(q)$, in liquidation under the pooling strategy. In this case, after the UI withdraws, both the FI $m$ and the FI $\ell$ liquidate $A_{I}$ and issue the same new security backed by $A_{O}$ to avoid the liquidation of $A_{O}$. Then, the repayment level is $\frac{I-L}{\sigma_{b}+\left(1-\sigma_{b}\right) \sigma_{Z}}$, which means that $\Pi_{F 2}^{P}(q)$ equals $\left[\sigma_{q}+\left(1-\sigma_{q}\right) \sigma_{Z}\right][Z-$ $\left.\frac{I-L}{\sigma_{b}+\left(1-\sigma_{b}\right) \sigma_{Z}}\right]$. We next examine the expected payoff of the $\mathrm{FI} \ell, \Pi_{F 2}^{S}(\ell)$, in liquidation under the separating strategy. In this case, if the FI $\ell$ liquidates $A_{I}$ and issues a new security backed by $A_{O}$ to avoid the liquidation of $A_{O}$, the outside investors demand $\frac{I-L}{\sigma_{\ell}+\left(1-\sigma_{\ell}\right) \sigma_{Z}}$ of the FI $\ell$ because they know the FI's type. Hence, $\Pi_{F 2}^{S}(\ell)$ equals $\left[\sigma_{\ell}+\left(1-\sigma_{\ell}\right) \sigma_{Z}\right] Z-I+L$. This implies that if the initial security is backed by $A_{I}$ and $A_{O}, \Pi_{F 2}^{S}(\ell)$ is smaller in continuation than in liquidation under separating, because $\sigma_{\ell}\left[R+Z-\frac{I}{\sigma_{\ell}+\left(1-\sigma_{\ell}\right) \sigma_{Z}}\right]+\left(1-\sigma_{\ell}\right) \sigma_{Z}[Z-$ $\left.\frac{I}{\sigma_{\ell}+\left(1-\sigma_{\ell}\right) \sigma_{Z}}\right]<\left[\sigma_{\ell}+\left(1-\sigma_{\ell}\right) \sigma_{Z}\right] Z-I+L$ under Assumption 1. Hence, if the separating strategy is possible, the FIm continues while the FI $\ell$ liquidates. Given these findings and $\Re_{1} \mathcal{L}-\left(\Re_{2}-\frac{1-\mathcal{L}}{\sigma_{\ell}}\right)>0$, we complete the proof by repeating the arguments of Lemma 2 .

Proof of Lemma 8: For $s=g$, the result of this lemma is trivial. For $s=b$, suppose that the UI withdraws. Then, the UI receives $I$ irrespective of whether the FI liquidates or continues. This is because the FI must avoid the liquidation of $A_{O}$ when liquidating $A_{I}$. On 
the other hand, suppose that the UI continues. Then, the interim expected payoff of the UI is $\sigma_{b} X+\left(1-\sigma_{b}\right) \sigma_{Z} X^{\prime}$. Hence, the UI continues if and only if $\sigma_{b} X+\left(1-\sigma_{b}\right) \sigma_{Z} X^{\prime} \geq I$.

Proofs of Lemma 9 and Proposition 2: Suppose that the FI offers an initial security backed by both $A_{I}$ and $A_{O}$. The discussions in Section 3.2 are modified as follows. First, given Lemma 7 , the partitions of the range of $\frac{R}{I}$ are (i) $\frac{1}{\sigma_{\ell}}>\frac{R}{I} \geq \Re_{1} \mathcal{L}$, (ii) $\Re_{1} \mathcal{L}>\frac{R}{I} \geq$ $\max \left(\Re_{2}-\frac{1-\mathcal{L}}{\sigma_{\ell}}, \frac{1}{\sigma_{m}}\right)$, and (iii) $\max \left(\Re_{2}-\frac{1-\mathcal{L}}{\sigma_{\ell}}, \frac{1}{\sigma_{m}}\right)>\frac{R}{I}>\frac{1}{\sigma_{m}}$. Second, Lemma 7 also implies that in the proof of Lemma 4, the objective functions of (P1) and (P3) are replaced by $\max _{X, X^{\prime}}$ $\Pi_{F 0}=\max _{X, X^{\prime}}\left[p+(1-p) \sigma_{b}\right](R-X+Z)+(1-p)\left(1-\sigma_{b}\right) \sigma_{Z}\left(Z-X^{\prime}\right)-(\bar{I}-I)$, whereas the objective functions of $(\mathrm{P} 4)$ and $(\mathrm{P} 5)$ are replaced by $\max _{X, X^{\prime}} \Pi_{F 0}=\max _{X, X^{\prime}} p(R-X+Z)$ $+(1-p)\left\{\delta \sigma_{m}\left(R+Z-Y^{m}\right)+\delta\left(1-\sigma_{m}\right) \sigma_{Z}\left(Z-Y^{m}\right)+(1-\delta)\left[\sigma_{\ell}+\left(1-\sigma_{\ell}\right) \sigma_{Z}\right](Z\right.$ $\left.\left.-Y^{\ell}\right)\right\}-(\bar{I}-I)$. Note that $Y^{m}$ and $Y^{\ell}$ in the latter are given by Lemma $7(\mathrm{ii})(\mathrm{b})(\beta)$ for each range of $\frac{R}{I}$. For the constraints of problems (P1)-(P5), (IRU1) is changed by $[p+(1-$ $\left.p) \sigma_{b}\right] X+(1-p)\left(1-\sigma_{b}\right) \sigma_{Z} X^{\prime} \geq I$, whereas (IRU4) is changed by $p X+(1-p) I \geq I$. It also follows from Lemma 8 that (ICU1) and (ICU3) are changed by $\sigma_{b} X+\left(1-\sigma_{b}\right) \sigma_{Z} X^{\prime} \geq$ $I$, whereas (ICU2) and (ICU4) are changed by $\sigma_{b} X+\left(1-\sigma_{b}\right) \sigma_{Z} X^{\prime}<I$. (NR) is changed by $R+Z \geq X \geq 0$ and $Z \geq X^{\prime} \geq 0$. For the remaining items in problems (P1)-(P5), there are no modifications.

Repeating arguments similar to those used to derive Lemma 4, we can derive the following findings. First, in the modified problems of (P1) and (P3), the optimal value of $\Pi_{F 0}$ is lower than $\widehat{\Pi}_{F 0}$ because the modified constraint of (ICU1) or (ICU3) is binding. The result depends on the fact that the UI obtains more if the modified constraint of (ICU1) or (ICU3) is binding than if the modified constraint of (IRU1) is binding. Second, in the modified problem (P2), the introduction of $X^{\prime}$ merely tightens the modified constraint of (ICU2). Thus, it is optimal to set $X^{\prime}=0$; that is, the initial security backed by $A_{I}$ alone is optimal. Third, in the modified problem $(\mathrm{P} 4)$, the requirement that the FI $\ell$ raises the additional amount $I-$ $L$ when liquidating $A_{I}$ causes the optimal value of $\Pi_{F 0}$ to be lower than $\Pi_{F 0}^{w}$ of (A12) in the original problem ( $\mathrm{P} 4)$. This is because the additional raising in liquidation tightens the self-selection constraint on the FI $\ell$ so that the FIm must issue the more underpriced security to separate itself from the FI $\ell$ (compare $Y^{m}$ in Lemma 2(ii) and $Y^{m}$ in the case of the higher $\frac{R}{I}$ in Lemma $\left.7(\mathrm{ii})(\mathrm{b})(\beta)\right)$. Indeed, the difference between the optimal values of the original and modified problems is given by $(1-p) \delta \frac{\sigma_{m}+\left(1-\sigma_{m}\right) \sigma_{Z}}{\sigma_{\ell}+\left(1-\sigma_{\ell}\right) \sigma_{Z}}(I-L) .{ }^{34}$ Fourth, in the modified

\footnotetext{
${ }^{34}$ Note that the occurrence of a decrease in $X$ due to an increase in the payoff of the UI under liquidation
} 
problem (P5), the optimal value of $\Pi_{F 0}$ equals $\Pi_{F 0}^{w}$ of (A13) in the original problem (P5) because the adverse selection issue is ineffective in this case (see $Y^{m}$ in Lemma 2(iii) and $Y^{m}$ in the case of the lower $\frac{R}{I}$ in Lemma $7($ ii $\left.)(\mathrm{b})(\beta)\right) .{ }^{35}$

Now, repeating arguments similar to those of Lemma 4, the configuration of the IFE under the initial security backed by both $A_{I}$ and $A_{O}$ is given by a form similar to the configuration of the IFEP, IFEC, and IFES of Lemma 4.

On the other hand, the maximization problems of the FI under uninformed finance are modified as follows. Using a procedure similar to that of Lemma 5, we can show that under uninformed finance, the FI continues (or liquidates) after the withdrawal of the UI if $\sigma_{b} R$ $-I \geq($ or $<)-(I-L)$; that is, $\sigma_{b} R \geq L\left(\right.$ or $\left.\sigma_{b} R<L\right)$. Note that the FI must raise the amount $I-L$ to avoid the liquidation of $A_{O}$ when liquidating $A_{I}$ regarding this security. Thus, we must analyze the two cases: (i) $\frac{1}{\sigma_{\ell}}>\frac{R}{I} \geq \max \left(\frac{\mathcal{L}}{\sigma_{b}}, \frac{1}{\sigma_{m}}\right)$, and (ii) $\max \left(\frac{\mathcal{L}}{\sigma_{b}}, \frac{1}{\sigma_{m}}\right)>$ $\frac{R}{I}>\frac{1}{\sigma_{m}}$. In case (i), the modified problem is the same as the modified problem of (P1) (or (P2)) under informed finance defined at the beginning of this proof if the FI induces the UI to continue (or withdraw), except that the FI does not pay $\epsilon_{I}$. In case (ii), the modified problem is the same as the modified problem of (P1) under informed finance defined at the beginning of this proof if the FI induces the UI to continue, except that the FI does not pay $\epsilon_{I}$. However, it is replaced by $\max _{X, X^{\prime}} p(R-X)+\omega Z+p I+(1-p) L-\bar{I}$ subject to $p X$ $+(1-p) I \geq I, \sigma_{b} X+\left(1-\sigma_{b}\right) \sigma_{Z} X^{\prime}<I, R+Z \geq X \geq 0$, and $Z \geq X^{\prime} \geq 0$ if the FI induces the UI to withdraw. Note that in this security, the UI can always recover $I$ even if it withdraws at time 1 . Now, repeating arguments similar to those used to derive Lemma 5 , we can show the result of Lemma 9.

Indeed, Lemma 6, with Lemmas 4, 5, and A3, shows that the optimal initial security backed by $A_{I}$ alone results in the optimal value of $\Pi_{F 0}$, which equals $\widehat{\Pi}_{F 0}$ under uninformed finance if $\frac{1}{\sigma_{\ell}}>\frac{R}{I} \geq \max \left(\phi(\mathcal{L}), \Re_{2}\right)$ or exceeds $\widehat{\Pi}_{F 0}$ under informed finance if $\max \left(\phi(\mathcal{L}), \Re_{2}\right)>$ $\left.\frac{R}{I}>\frac{1}{\sigma_{m}}\right)$. The optimal value of $\Pi_{F 0}$ in Lemma 6 is also continuous even at the threshold lines dividing the $\left(\mathcal{L}, \frac{R}{I}\right)$ space into each region. However, if the FI offers an initial security backed by $A_{I}$ and $A_{O}$, under informed finance, the optimal value of $\Pi_{F 0}$ in each of the modified problems is lower than or equal to that of the corresponding original problem; further, under uninformed finance, the optimal value of $\Pi_{F 0}$ is equal to $\widehat{\Pi}_{F 0}$ if $\frac{R}{I} \geq \frac{\mathcal{L}}{\sigma_{b}}\left(<\frac{1}{\sigma_{b}}\right)$, and is lower than or equal to $\widehat{\Pi}_{F 0}$ if $\frac{\mathcal{L}}{\sigma_{b}}>\frac{R}{I}$. Thus, we establish the result of Proposition 2 .

(see the modified constraint of (IRU4)) cancels out the additional payment of $Y^{\ell}$ given by Lemma $7(\mathrm{ii})(\mathrm{b})(\beta)$. ${ }^{35}$ Again, a decrease in $X$ cancels out the additional payment of $Y^{\ell}$. 
Proof of Proposition 3: The results of $\frac{R}{I}$ and $\mathcal{L}$ are evident from Lemma 6 and $\phi^{\prime}(\mathcal{L})>$ 0. In addition, $\frac{\partial \Re_{2}}{\partial \sigma_{m}}<0, \frac{\partial \Re_{2}}{\partial \sigma_{\ell}}<0, \frac{\partial \Re_{2}}{\partial \sigma_{Z}}>0, \frac{\partial \phi(0)}{\partial \sigma_{m}}<0, \frac{\partial \phi(0)}{\partial \sigma_{\ell}}<0, \frac{\partial \phi(0)}{\partial \sigma_{Z}}>0, \frac{\partial \phi^{\prime}(\mathcal{L})}{\partial \sigma_{m}}<0, \frac{\partial \phi^{\prime}(\mathcal{L})}{\partial \sigma_{\ell}}$ $<0$ and $\frac{\partial \phi^{\prime}(\mathcal{L})}{\partial \sigma_{Z}}>0$. Given $\phi^{\prime}(\mathcal{L})>0$ and $\phi(1)=\Re_{1}$, the results of $\sigma_{m}, \sigma_{\ell}$ and $\sigma_{Z}$ are derived from comparative statics on the condition in Lemma 6(i).

Optimality of the short-term debt (Section 6.1): Note that the FI always chooses to continue if the UI cannot withdraw at time 1 . Then, the optimal security design problem for the FI at time 0 when the initial security is backed by the cash flow of $A_{I}$ is: $\max _{X_{F}} \Pi_{F 0}$ given by (P1), subject to (IRU1) and (NR). Solving this problem, we can show that the ex ante expected payoff of the FI is equal to $\widehat{\Pi}_{F 0}$. Similarly, in the case of the initial security backed by the cash flow of both $A_{I}$ and $A_{O}$, we can prove that the ex ante expected payoff of the FI is equal to $\widehat{\Pi}_{F 0}$.

The participation of the UI in the capital market at time 1: If the UI can buy the security issued by the FI in the capital market at time 1, the results of Sections 3.1 .1 and 3.1.2 still hold. However, the results of Section 3.1.3 must be modified if the UI obtains a positive rent in so doing. Hence, using Lemma 2, Lemma 3 is rewritten as follows.

Lemma $3^{\prime}$ : Suppose that $s=b$.

(i) If $\Re_{1}>\frac{R}{I} \geq \Re_{2}$, the UI continues if $\sigma_{b} X \geq \frac{\delta\left[\sigma_{m}+\left(1-\sigma_{m}\right) \sigma_{Z}\right] \sigma_{\ell} R}{\sigma_{\ell}+\left(1-\sigma_{\ell}\right) \sigma_{Z}}+(1-\delta) L$. Otherwise, the UI withdraws.

(ii) If $\frac{R}{I} \geq \Re_{1}$ or if $\frac{R}{I}>\Re_{2}$, the results of Lemma 3 still hold.

Using Lemma $3^{\prime}$, we must rearrange (ICU3) and (ICU4) in problems (P3) and (P4) when $\Re_{1}>\frac{R}{I} \geq \Re_{2}$. We must also rearrange (IRU4) in problem (P4) when $\Re_{1}>\frac{R}{I} \geq \Re_{2}$, because the reservation utility of the UI increases by the same amount as the expected positive rent. For the other items in problems (P1)-(P5) or for the uninformed case, there are no modifications. Thus, in problems (P3) and (P4), this extension tightens (ICU3) and (IRU4) but relaxes (ICU4). In the equilibrium given by Lemmas 4 and 6, only (IRU4) is binding at the solution to problem $(\mathrm{P} 4)$. On the other hand, in the equilibrium given by Lemma 6 , the solution to problem (P3) is dominated. These findings show that this extension merely expands the UFE region of Lemma 6(i), but reduces the IFES region of Lemma 6(ii) where the FIm repays outside investors' premiums, because the FI must increase $X$ in the IFES.

Applying similar arguments to obtain the results corresponding to Lemmas 7-9, we can derive results similar to those of Propositions 1 and 2.

Relaxation of Assumption 4: If Assumption 4 is not satisfied, $Z \geq Y$ must be binding in 
the proof of Lemma 2. Then, the monotonicity constraint is satisfied with strict inequality: $Y_{R+Z}>Y_{Z}$. In fact, even if $Y_{R+Z}^{P}>Y_{Z}^{P}$ in Lemma 2(i), this does not affect the result of Lemma 4(i) under the risk-neutrality assumption. On the other hand, if $Y_{R+Z}^{m}>Y_{Z}^{m}$ in Lemmas 2(ii) and (iii), this effect makes it more likely that the FI $\ell$ will mimic the FIm because the most effective security design for deterring the FI $\ell$ from mimicking the FIm is that $Y_{R+Z}^{m}=Y_{Z}^{m}=Y^{m}$. Hence, the relaxation of Assumption 4 decreases the ex ante expected payoff of the FI if the UI really withdraws at $s=b$ and if the ex post equilibrium in the capital market is separating. This implies that the combined region of the IFEP and IFEC of Lemmas 4(i) and (ii) and the UFE region of Lemma 6(i) expand. However, except for these changes, the main results of Lemmas 4 and 6 (that is, Proposition 1 ) are preserved. If $\max \left[\frac{\sigma_{\ell} R}{\sigma_{\ell}+\left(1-\sigma_{\ell}\right) \sigma_{Z}}, \frac{I}{\sigma_{\ell}+\left(1-\sigma_{\ell}\right) \sigma_{Z}}\right]>Z \geq \frac{I-L}{\sigma_{\ell}+\left(1-\sigma_{\ell}\right) \sigma_{Z}}$, the FI can avoid the liquidation of $A_{O}$ when liquidating $A_{I}$. Then, Proposition 2 still holds, because with $Z \geq Y$ binding, the advantage of the initial security backed by both $A_{I}$ and $A_{O}$ is reduced. If $\frac{I-L}{\sigma_{\ell}+\left(1-\sigma_{\ell}\right) \sigma_{Z}}>Z$ $>0$, the FI $\ell$ prefers to liquidate $A_{O}$ when liquidating $A_{I}$. However, as long as the expected cash flow of $A_{O}$ at $s=b$ is larger than the liquidation value of $A_{O}(\equiv 0)$, the advantage of the initial security backed by both $A_{I}$ and $A_{O}$ is further reduced. Hence, Proposition 2 continues to hold. Furthermore, whichever happens, the optimality of the new security backed by both $A_{I}$ and $A_{O}$ at time 1 also continues to hold because the difference between the bankruptcy losses of the FI $\ell$ and the FI $m$ is still the largest in this class of security.

Relaxation of Assumption 5: To simplify the analysis, we focus on the case in which the FI can choose whether to undertake information acquisition. Given the proofs of Lemmas 4 and 6, Assumption 5(i) ensures that only (ICU3) (or (IRU4)) binds in problem (P3) (or (P4) and (P5)), that $X^{w}<R$ holds in problems (P4) and (P5), and that ( $\Pi_{F 0}^{*}$ of Lemma $5(\mathrm{i}))>\left(\Pi_{F 0}^{*}\right.$ of Lemma A3(ii)(a)). Without Assumption 5(i), we obtain the following result.

Lemma A4: Suppose that Assumption 5(i) is not satisfied: $\delta \leq 1-\frac{\sigma_{m}}{\sigma_{m}-\sigma_{\ell}} \frac{p\left(1-\sigma_{b}\right)}{p+(1-p) \sigma_{b}}$.

(i) If $\mathcal{L}>\frac{\sigma_{b}-\delta\left[p+(1-p) \sigma_{b}\right]}{(1-\delta)\left[p+(1-p) \sigma_{b}\right]}$ and $\frac{R}{I} \geq \frac{1-(1-p)[\delta+(1-\delta) \mathcal{L}]}{p}$, the result of Lemma 6 still holds.

(ii) Suppose that $\mathcal{L}>\frac{\sigma_{b}-\delta\left[p+(1-p) \sigma_{b}\right]}{(1-\delta)\left[p+(1-p) \sigma_{b}\right]}$ and $\frac{R}{I}<\frac{1-(1-p)[\delta+(1-\delta) \mathcal{L}]}{p}$ or that $\mathcal{L} \leq \frac{\sigma_{b}-\delta\left[p+(1-p) \sigma_{b}\right]}{(1-\delta)\left[p+(1-p) \sigma_{b}\right]}$.

(a) If $\frac{1}{\sigma_{\ell}}>\frac{R}{I} \geq \frac{1}{\sigma_{b}}$, the equilibrium is given by the UFE that is featured by Lemma 5(i). (b) If $\frac{1}{\sigma_{b}}>\frac{R}{I}>\frac{1}{\sigma_{m}}$, the equilibrium is given by the UFE that is featured in Lemma $5($ ii) or the IFE in which the FI induces the UI to continue.

Proof of Lemma A4: If $\mathcal{L}>\frac{\sigma_{b}-\delta\left[p+(1-p) \sigma_{b}\right]}{(1-\delta)\left[p+(1-p) \sigma_{b}\right]}$ and $\frac{R}{I} \geq \frac{1-(1-p)[\delta+(1-\delta) \mathcal{L}]}{p}$, we can still ensure that only (ICU3) (or (IRU4)) binds in problem (P3) (or (P4) and (P5)), that $X^{w} \leq R$ holds 
in problems $(\mathrm{P} 4)$ and $(\mathrm{P} 5)$, and that $\left(\Pi_{F 0}^{*}\right.$ of Lemma $\left.5(\mathrm{i})\right)>\left(\Pi_{F 0}^{*}\right.$ of Lemma A3(ii)(a)). However, if $\mathcal{L}>\frac{\sigma_{b}-\delta\left[p+(1-p) \sigma_{b}\right]}{(1-\delta)\left[p+(1-p) \sigma_{b}\right]}$ and $\frac{R}{I}<\frac{1-(1-p)[\delta+(1-\delta) \mathcal{L}]}{p}$, (IRU4) is inconsistent with $X \leq R$ in problems (P4) and (P5). Then, in the range $\Re_{1}>\frac{R}{I}$ under informed finance, the optimal initial security is given by that which induces the UI to continue and sets (ICU3) to be binding in problem (P3). However, this implies that the optimal initial security generates $\Pi_{F 0}^{*}$ of Lemma A3(ii)(a). Given that $\left(\Pi_{F 0}^{*}\right.$ of Lemma $\left.5(\mathrm{i})\right)>\left(\Pi_{F 0}^{*}\right.$ of Lemma A3(ii)(a)) in this range and that $\Re_{2}>\frac{1}{\sigma_{b}}$, the IFE is dominated by the UFE of Lemma 5 (i) if $\frac{1}{\sigma_{\ell}}>\frac{R}{I}$ $\geq \frac{1}{\sigma_{b}}$. Now, if $\mathcal{L} \leq \frac{\sigma_{b}-\delta\left[p+(1-p) \sigma_{b}\right]}{(1-\delta)\left[p+(1-p) \sigma_{b}\right]}$, we can show that in the range of $\Re_{1}>\frac{R}{I}$, the only IFE is given by the optimal initial security that induces the UI to continue and sets (IRU1) to be binding in problem (P3). Furthermore, we can prove that $\Pi_{F 0}^{*}$ of this IFE is the same as that of the UFE of Lemma 5 (i) if $\frac{1}{\sigma_{\ell}}>\frac{R}{I} \geq \frac{1}{\sigma_{b}}$, except that the FI must pay $\epsilon_{I}$ in the IFE. $\|$

Hence, even though Assumption 5(i) is not satisfied, the main result of Lemma 6 or Proposition 1 is preserved if $\frac{R}{I}$ and $\mathcal{L}$ are large enough; it also holds for $\frac{1}{\sigma_{\ell}}>\frac{R}{I} \geq \frac{1}{\sigma_{b}}$ if $\mathcal{L}$ is not large enough or if $\mathcal{L}$ is large enough although $\frac{R}{I}$ is not large enough. In this region, Proposition 2 also holds.

Next, if Assumption 5(ii) is not satisfied, then $\frac{1}{\sigma_{b}} \geq \Re_{2}$ (however, note that $\Re_{1}>\frac{1}{\sigma_{b}}$ ). Thus, from Lemmas 4 and 5, we obtain the following result instead of Lemma 6.

Lemma A5. Suppose that Assumption 5(ii) is not satisfied: $\delta \leq \frac{\left(1-\sigma_{Z}\right) \sigma_{\ell}}{\sigma_{\ell}+\left(1-\sigma_{\ell}\right) \sigma_{Z}}$. (i) If $\frac{1}{\sigma_{\ell}}$ $>\frac{R}{I} \geq \max \left(\phi(\mathcal{L}), \frac{1}{\sigma_{b}}\right)$, the equilibrium is given by the UFE that is featured by Lemma $5(i)$. (ii) If $\max \left(\phi(\mathcal{L}), \frac{1}{\sigma_{b}}\right)>\frac{R}{I} \geq \max \left(\phi(\mathcal{L}), \Re_{2}\right)$, the equilibrium is given by the IFES that is featured by Lemma 6(ii) where the FIm repays outside investors' premiums or the UFE that is featured by Lemma 5(ii). (iii) If $\max \left(\phi(\mathcal{L}), \Re_{2}\right)>\frac{R}{I}>\frac{1}{\sigma_{m}}$, the result of Lemma 6 still holds.

The only difference is that if $\frac{R}{I}$ lies in the middle range of $\frac{R}{I} \in\left(\Re_{2}, \frac{1}{\sigma_{b}}\right)$, there may be a UFE region where both the UI and the FI continue at $s=b$ or a UFE region where the UI withdraws and the FI liquidates $A_{I}$ at $s=b$. Thus, in this range, the overinvestment problem may occur because the UI does not withdraw at $s=b$, or the underinvestment problem may arise because overliquidation cannot be prevented. Further, Proposition 2 holds in any $\frac{R}{I} \in$ $\left(\frac{1}{\sigma_{h}}, \frac{1}{\sigma_{\ell}}\right)$ outside of this middle range. 


\section{References}

Acharya, V.V., Viswanathan, S., 2011. Leverage, moral hazard, and liquidity. Journal of Finance 66(1), 99-138.

Ayotte, K., Gaon, S., 2011. Asset-backed securities: costs and benefits of "bankruptcy remorteness". Review of Financial Studies 24(4), 1299-1336.

Axelson, U., 2007. Security design with investor private information. Journal of Finance $62(6), 2587-2632$.

Axelson, U., Stromberg, P., Weisbach, M. S., 2009. Why are buyouts leveraged: the financial structure of private equity funds. Journal of Finance 64(4), 1549-1582.

Banks, J.S., Sobel, J., 1987. Equilibrium selection in signaling games. Econometrica 55(3), 647-661.

BearStearns., 2007. Asset-backed commercial paper (ABCP) conduits and SIVs: what are the issues? Structured Credit Research August 9.

Benveniste, L.M., Berger, A.N., 1987. Securitization with recourse: an instrument that offers uninsured bank depositors sequential claims. Journal of Banking and Finance 11(3), 403-424.

Berglof, E., von Thadden, E.-L., 1994. Short-term versus long-term interests: capital structure with multiple investors. Quarterly Journal of Economics 109(4), 1055-1084.

Bolton, P., Freixas, X., 2000. Equity, bonds, and bank debt: capital structure and financial market equilibrium under asymmetric information. Journal of Political Economy 108(2), 324-351.

Bolton, P., Santos, T., Scheinkman, J.A., 2011. Outside and inside liquidity. Quarterly Journal of Economics 126(1) 259-321.

Boot, A.W.A., Greenbaum, S.I., Thakor A.V., 1993. Reputation and discretion in financial contracting. American Economic Review 83(5), 1165-1183.

Brunnermeier, M.K., 2009. Deciphering the liquidity and credit crunch 2007-08. Journal of Economic Perspectives 23(1), 77-100.

Brunnermeier, M.K., Oehmke, M., 2010. The maturity rat race. NBER Working Paper 16607.

Calomiris, C.W., Kahn, C.M., 1991. The role of demandable debt in structuring optimal banking arrangements. American Economic Review 81(3), 497-513.

Campello, M., De Matta, R.A., 2011. Credit default swaps, firm financing and the economy. SSRN Working Paper, 1770066.

Carlstrom, C. T., Samolyk, K. A., 1995. Loan sales as a response to market-based capital constraints. Journal of Banking and Finance 19(3-4), 627-646.

Chemmanur, T.J., John, K., 1996. Optimal incorporation, structure of debt contracts, and limited-recourse project financing. Journal of Financial Intermediation 5(4), 372-408.

Coval, J., Jurek, J., Stafford, E.. 2009. The economics of structured finance. Journal of 
Economic Perspectives 23(1), 3-25.

DeMarzo, P. M., 2005. The pooling and tranching of securities: a model of informed intermediation. Review of Financial Studies 18(1), 1-35.

DeMarzo, P. M., Duffie, D., 1999. A liquidity-based model of security design. Econometrica $67(1), 65-99$.

Diamond, D. W., Rajan, R. G., 2001. Liquidity risk, liquidity creation, and financial fragility: a theory of banking. Journal of Political Economy 109(2), 287-327.

Diamond, D. W., Raghuram R. G., 2011. Fear of fire sales, illiquidity seeking, and the credit freeze. Quarterly Journal of Economics, 126(2), 557-591.

FitchRatings, 2007. Asset-backed commercial paper \& global banks exposure -10 key questions. Special Report September 12.

Flannery, M. J., 1994. Debt maturity and the deadweight cost of leverage: optimally financing banking firms. American Economic Review 84(1), 320-331.

Glaeser, E. L., Kallal, H. D., 1997. Thin markets, asymmetric information, and mortgagebacked securities. Journal of Financial Intermediation 6(1), 64-86.

Gorton, G. B., 2009. The Subprime Panic. European Financial Management 15(1), 10-46.

Gorton, G. B., Souleles, N., 2006. Special purpose vehicles and securitization. in Stultz, R., Caray, M., eds. The Risk of Financial Institutions Chicago: Chicago University Press for NBER.

He, Z., and Xiong, W., 2009. Dynamic debt runs. Review of Financial Studies, forthcoming. Holmström, B., Tirole J., 1998. Private and public supply of liquidity. Journal of Political Economy 106(1), 1-40.

Huang, R., Ratnovski, L., 2011. The dark side of bank wholesale funding Journal of Financial Intermediation 20(2), 248-263.

IMF. October, 2007. Financial market turbulence: causes, consequences, and policies. Global Financial Stability Report.

IMF. December, 2007. Subprime: tentacles of a crisis. Finance and Development.

Inderst, R., Mueller, H. M., 2010. CEO replacement under private information. Review of Financial Studies 23(8), 2935-2969.

Innes, R. D. 1990. Limited liability and incentive contracting with ex ante action choices. Journal of Economic Theory 52(1), 45-67.

John, T., 1993. Optimality of spin-offs and allocation of debt. Journal of Financial and Quantitative Analysis 28(1), 139-160.

Jones, D., 2000. Emerging problems with the Basel capital accord: regulatory capital arbitrage and related issues. Journal of Banking and Finance 24(1), 35-58.

Kahn, C., Winton, A., 2004. Moral hazard and optimal subsidiary structure for financial institutions. Journal of Finance 59(6), 2531-2575. 
Keys, B. J., Mukherjee, T., Seru, A., Vig, V., 2009. Financial regulation and securitization: evidence from Subprime loans. Journal of Monetary Economics 56(5), 700-720.

Nachman, D. C., Noe, T. H., 1994. Optimal design of securities under asymmetric information. Review of Financial Studies 7(1), 1-44.

Repullo, R., Suarez, J., 1998. Monitoring, liquidation, and security design. Review of Financial Studies 11(1), 163-187.

Riddiough, T. J. 1997. Optimal design and governance of asset-backed securities. Journal of Financial Intermediation 6(2), 121-152.

Segura, A., Suarez, J., 2011. Liquidity shocks, roll-over risk and debt maturity. CEPR Discussion Paper 8324.

Shleifer, A., Vishny, R. W., 2010. Unstable banking. Journal of Financial Economics 97(3), 306-318.

Stein, J. C., 2005. Why are most funds open-end? Competition and the limits of arbitrage. Quarterly Journal of Economics 120(1), 247-272.

Uhlig, H., 2010. A model of a systemic bank run. Journal of Monetary Economics 57(1), 78-96.

Winton, A., 1995. Costly state verification and multiple investors: the role of seniority. Review of Financial Studies 8(1), 91-123.

Winton, A., 2003. Institutional liquidity needs and the structure of monitored finance. Review of Financial Studies 16(4), 1273-1313. 


\begin{tabular}{|c|c|c|c|c|c|}
\hline state of & \multicolumn{2}{|c|}{ asset quality of $A_{I}$} & \multicolumn{3}{|c|}{ cash flows of $A_{I}$ and $A_{O}$} \\
\hline$s$ & $q$ & probability & $\begin{array}{c}\text { cash flow } \\
\text { of } A_{I}\end{array}$ & $\begin{array}{c}\text { cash flow } \\
\text { of } A_{O}\end{array}$ & $\begin{array}{c}\text { conditional } \\
\text { probability } \\
\text { on } q\end{array}$ \\
\hline$g$ & $h$ & $p$ & $R$ & Z & 1 \\
\hline \multirow[t]{2}{*}{$b$} & $m$ & $(1-p) \delta$ & $\begin{array}{l}R \\
0 \\
0\end{array}$ & $\begin{array}{l}Z \\
Z \\
0\end{array}$ & $\begin{array}{c}\sigma_{m} \\
\left(1-\sigma_{m}\right) \sigma_{Z} \\
\left(1-\sigma_{m}\right)\left(1-\sigma_{Z}\right)\end{array}$ \\
\hline & $\ell$ & $(1-p)(1-\delta)$ & $\begin{array}{l}R \\
0 \\
0\end{array}$ & $\begin{array}{l}Z \\
Z \\
0\end{array}$ & $\begin{array}{c}\sigma_{\ell} \\
\left(1-\sigma_{\ell}\right) \sigma_{Z} \\
\left(1-\sigma_{\ell}\right)\left(1-\sigma_{Z}\right)\end{array}$ \\
\hline
\end{tabular}

Table 1. The cash flows of $A_{1}$ and $A_{0}$ and their probabilities conditional on $q$. 
$t=0$

FI’s initial
security offer

FI’s initial
security offer

(s, q)

realized

$t=1$

UI's

continuation

/withdrawal

decision

FI's

continuation

/liquidation

decision

$t=2$

Cash flow

realized

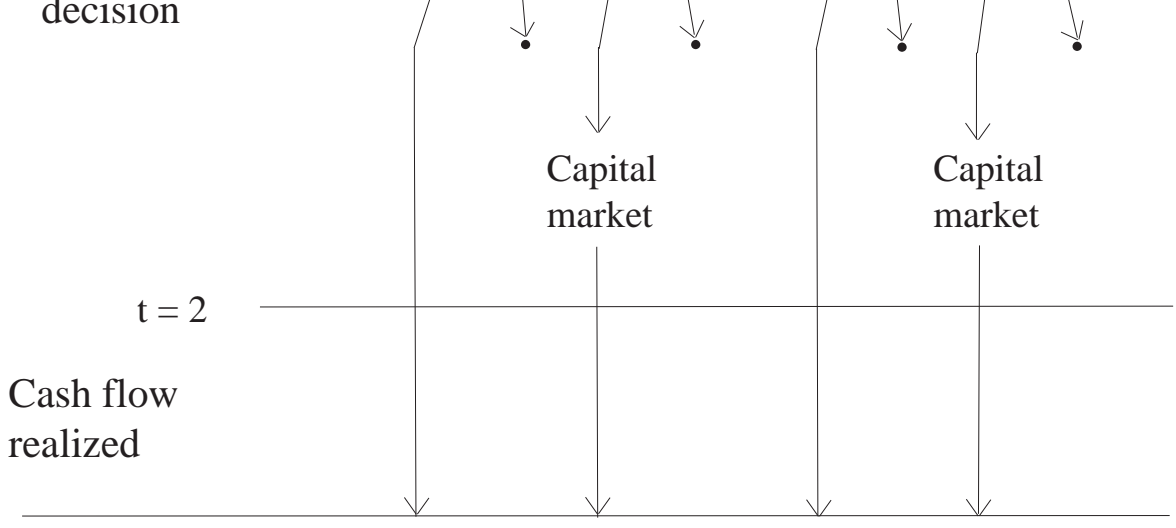

Figure 1. Timeline in the Basic Model. C: continuation; W: withdrawal; and L: liquidation. 


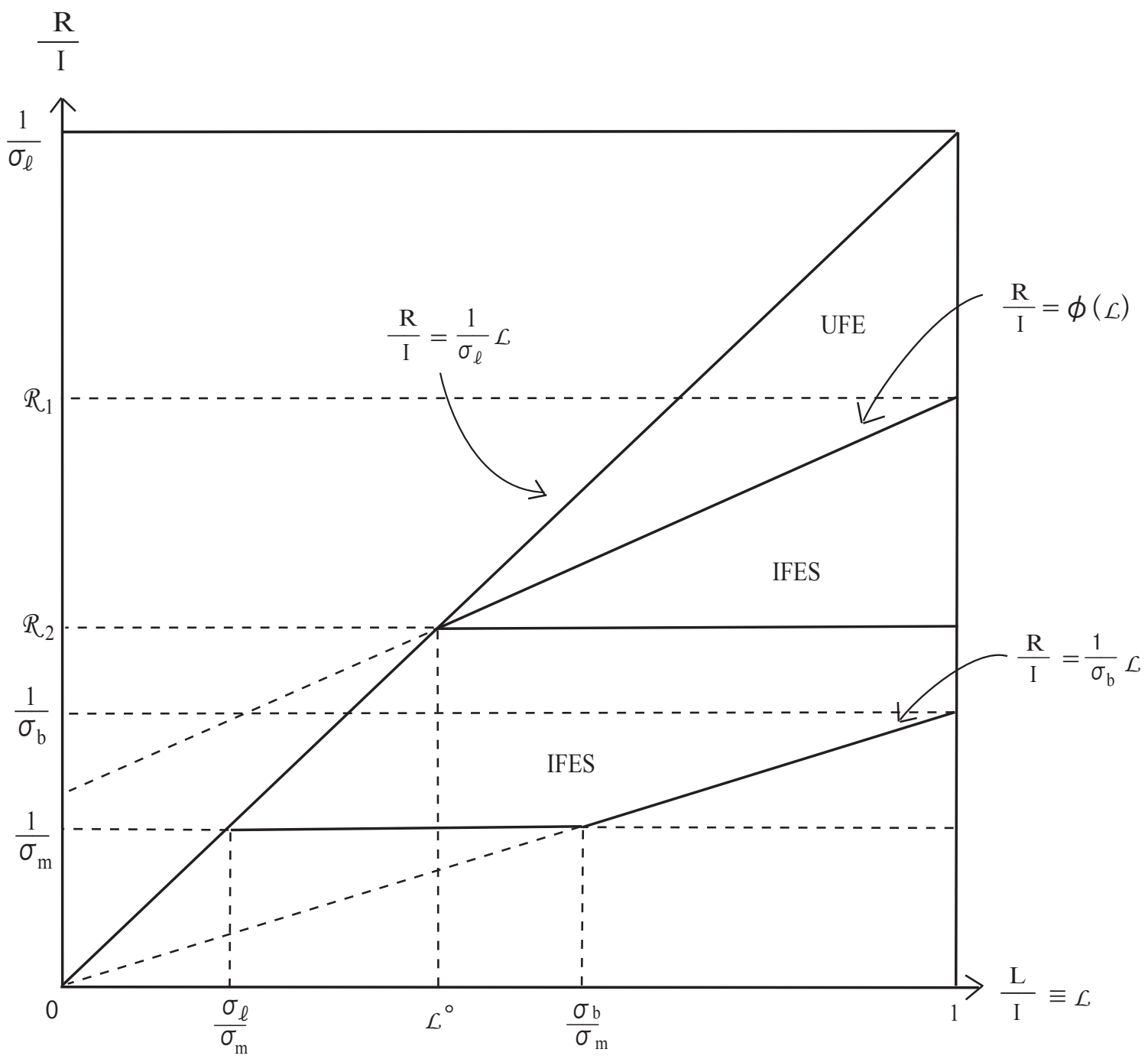

Figure 2. The configuration of equilibrium. The straight lines $\frac{\mathrm{R}}{\mathrm{I}}=\frac{1}{\sigma_{\ell}} \mathcal{L}$ and $\frac{\mathrm{R}}{\mathrm{I}}=\frac{1}{\sigma_{\mathrm{b}}} \mathcal{L}$ follow from $\sigma_{\mathrm{b}} \equiv \delta \sigma_{\mathrm{m}}+(1-\delta) \sigma_{\ell}$ and the assumption that $\sigma_{\ell} \mathrm{R}<\mathrm{L}<\sigma_{\mathrm{b}} \mathrm{R}$. Note that $\mathbb{R}_{1} \equiv \frac{\sigma_{\ell}+\left(1-\sigma_{\ell}\right) \sigma_{\mathrm{z}}}{\sigma_{\ell}\left[\sigma_{\mathrm{b}}+\left(1-\sigma_{\mathrm{b}}\right) \sigma_{\mathrm{z}}\right]}, \mathbb{R}_{2} \equiv \frac{\sigma_{\ell}+\left(1-\sigma_{\ell}\right) \sigma_{\mathrm{z}}}{\sigma_{\ell}\left[\sigma_{\mathrm{m}}+\left(1-\sigma_{\mathrm{m}}\right) \sigma_{\mathrm{z}}\right]}$, $\phi(\mathcal{L}) \equiv[\delta+(1-\delta) \mathcal{L}] \mathbb{R}_{1}, \quad \stackrel{\circ}{\mathcal{L}} \equiv \frac{\sigma_{\ell}+\left(1-\sigma_{\ell}\right) \sigma_{\mathrm{z}}}{\sigma_{\mathrm{m}}+\left(1-\sigma_{\mathrm{m}}\right) \sigma_{\mathrm{z}}}$, and $\phi\left(\mathcal{L}^{\circ}\right)=\mathbb{R}_{2}$. 